JOURNAL OF THE

AMERICAN MATHEMATICAL SOCIETY

Volume 13, Number 2, Pages 371-410

S 0894-0347(00)00326-X

Article electronically published on January 31, 2000

\title{
THE ENUMERATIVE GEOMETRY OF $K 3$ SURFACES AND MODULAR FORMS
}

\author{
JIM BRYAN AND NAICHUNG CONAN LEUNG
}

\section{Contents}

1. Introduction 371

2. Invariants of families of symplectic structures

3. Twistor families of $K 3$ surfaces and the definition of $N_{g}(n) \quad 378$

4. Computation of $N_{g}(n) \quad 381$

5. Analysis of moduli spaces and local contributions

5.1. Components of $\overline{\mathcal{M}}_{\mathbf{a}, \mathbf{b}}$

5.2. Obstruction theory and virtual classes

5.3. Computations via blow-ups on $\mathbf{P}^{2} \quad 396$

6. Counting curves on the rational elliptic surface

Appendix A. Virtual classes, point constraints, and base changes 401

$\begin{array}{lll}\text { A.1. Base changes for perfect relative obstruction theories } & 402\end{array}$

A.2. Virtual classes and point constraints 404

Appendix B. A deformation result 406

References $\quad 409$

\section{InTRODUCTION}

Let $X$ be a $K 3$ surface, and let $C$ be a holomorphic curve in $X$ representing a primitive homology class. For any $g$ and $n$ satisfying $C^{2} / 2=g+n-1$, we define an invariant $N_{g}(n)$ which counts the number of curves of geometric genus $g$ with $n$ nodes passing through $g$ points in $X$ in the linear system $|C| . N_{g}(n)$ is well defined for any $(X, C)$ and is invariant under those deformations of the Kähler structure on $X$ that preserve the $(1,1)$-type of the class $[C]$. For a generic $X$ and generic choices of the $g$ points, $N_{g}(n)$ is enumerative, i.e. it is precisely the number of genus $g$ curves passing through $g$ points in the class $[C]$ (Theorem 3.5). For each $g$, consider the generating function

$$
F_{g}(q)=\sum_{n=0}^{\infty} N_{g}(n) q^{g+n-1} .
$$

Our main theorem gives explicit formulas for $F_{g}$ in terms of quasi-modular forms:

Received by the editors January 5, 1998 and, in revised form, October 18, 1999.

2000 Mathematics Subject Classification. Primary 14N35, 53D45, 14J28.

The first author is supported by a Sloan Foundation Fellowship and NSF grant DMS-9802612 and the second author is supported by NSF grant DMS-9626689.

(C)2000 American Mathematical Society 
Theorem 1.1 (Main Theorem). For any $g$, we have

$$
\begin{aligned}
F_{g}(q) & =\left(\sum_{k=1}^{\infty} k\left(\sum_{d \mid k} d\right) q^{k}\right)^{g} q^{-1} \prod_{m=1}^{\infty}\left(1-q^{m}\right)^{-24} \\
& =\frac{\left(D G_{2}\right)^{g}}{\Delta}
\end{aligned}
$$

where $D=q \frac{d}{d q}$ is logarithmic differentiation, $G_{2}$ is the Eisenstein series, and $\Delta$ is the discriminant.

So, for example, we have

$$
\begin{aligned}
& F_{0}=q^{-1}+24+324 q^{1}+3200 q^{2}+\cdots, \\
& F_{1}=1+30 q+480 q^{2}+5460 q^{3}+\cdots, \\
& F_{2}=q+36 q^{2}+672 q^{3}+8728 q^{4}+\cdots, \\
& F_{3}=q^{2}+42 q^{3}+900 q^{4}+13220 q^{5}+\cdots .
\end{aligned}
$$

If we write $q=e^{2 \pi i \tau}$, then $\Delta(\tau)=q \prod_{m=1}^{\infty}\left(1-q^{m}\right)^{24}=\eta(\tau)^{24}$ is a modular form of weight 12 where $\eta(\tau)$ is the Dedekind $\eta$ function. In particular, for any $\left(\begin{array}{ll}a & b \\ c & d\end{array}\right) \in S L(2, \mathbf{Z})$ and $\operatorname{Im}(\tau)>0$, we have

$$
\Delta\left(\frac{a \tau+b}{c \tau+d}\right)=(c \tau+d)^{12} \Delta(\tau) .
$$

$G_{2}(q)$ is the Eisenstein series

$$
G_{2}(q)=\frac{-1}{24}+\sum_{k=1}^{\infty} \sigma(k) q^{k}
$$

where $\sigma(k)=\sum_{d \mid k} d . G_{2}$ and its $D$ derivatives are quasi-modular forms [18]. Quasimodular forms are closed under multiplication and $D$, so, in particular, $\left(D G_{2}\right)^{g} \Delta^{-1}$ is a quasi-modular form.

When $g=0$, our main theorem proves the formula predicted by Yau and Zaslow 37. for primitive classes. For $g \geq 0$, Göttsche has recently conjectured a very general set of formulas for the number of curves on algebraic surfaces [18] and Theorem 1.1 proves his conjecture for primitive classes in $K 3$ surfaces. Yau and Zaslow give a beautiful, though indirect, argument that was completed into proof for the $g=0$ case by Beauville [3] under the assumption that all the curves in $|C|$ are reduced and irreducible and under the understanding that one should count curves with certain positive integral multiplicities. The precise nature of these multiplicities and their relationship to stable maps was explained by Fantechi-Göttsche-van Straten [15. One expects that for generic choices, all the curves will be nodal and the multiplicities will all be one; Chen [10] has partial results along this line 1

We shall use a completely different argument by studying Gromov-Witten invariants for the twistor family of symplectic structures on a $K 3$ surface. We learned the twistor family approach from Li and Liu 29] who studied the Seiberg-Witten theory for families and obtained interesting results.

\footnotetext{
${ }^{1}$ As this paper was going through its final revisions, Chen completed his program; his paper 11] shows that for a generic $K 3$ all the rational curves in a primitive, ample linear system are nodal.
} 
In the case of a hyperkähler $K 3$, the twistor family is the unit sphere in the space of self-dual harmonic 2-forms. The idea of extending the moduli space of pseudoholomorphic curves by including the family of non-degenerate, norm 1, self-dual, harmonic 2-forms goes back to Donaldson [14]. He pointed out that in order to have the theory of pseudo-holomorphic curves on a 4-manifold more closely mimic the theory of divisors on a projective surface, one should include this family.

One key point in the proof of our main theorem is the use of the large diffeomorphism group of a $K 3$ surface to move $C$ to a particular class $S+(g+n) F$ on an elliptic $K 3$ surface with section $S$ and fiber $F$ which has 24 nodal fibers. Inside the linear system $|S+(g+n) F|$, we can completely understand the moduli space of stable maps and directly compute the invariants $N_{g}(n)$, reducing the calculation to the computation of "local contributions" by multiple covers.

The contribution of multiple covers of the smooth fibers is responsible for the $D G_{2}$ term and the contribution from multiple covers of the nodal fibers is related to the partition function $p(d)$. The computation for the multiple covers of nodal fibers requires a virtual class computation. This is done by first splitting the moduli space into components and then identifying each component with product of moduli spaces and show that the virtual class also splits as a product. Each factor is then identified with a moduli-obstruction problem arising from the Gromov-Witten invariants of a certain blow-up of $\mathbf{P}^{2}$. This "matching" technique allows us to use known properties of the Gromov-Witten invariants of $\mathbf{P}^{2}$ blown up, specifically their invariance under Cremona transformations, to show that the contribution of each component is always 0 or 1 . The partition function then arises combinatorially in a somewhat unusual way (see Lemma [5.9). These computations occupy section [5]

We prove that the invariants $N_{g}(n)$ are enumerative. Our proof shows that for generic choices of $X$ and the points, our invariant enumerates curves of geometric genus $g$ on $X$ counting each curve with a positive integral multiplicity that is one when all the singularities are nodal (Theorem 3.5). Utilizing Chen's recent result [11] we can further conclude that all the curves are in fact nodal and so the invariant is enumerative in the strongest sense.

We can also apply our method to certain rational surfaces. We blow up $\mathbf{P}^{2}$ at nine distinct points and call the resulting algebraic surface $Y$. We consider the Gromov-Witten invariant $N_{g}^{Y}(C)$ which counts the number of curves of geometric genus $g$ passing through $g$ generic points in a fixed class $C$. We show that any class $C \in H_{2}(Y)$ whose genus $g$ Gromov-Witten invariants require exactly $g$ point constraints is related to a class of the form $C_{n}=(g+n)\left[3 h-\sum_{i=1}^{9} e_{i}\right]+e_{9}$ by a Cremona transform. Here $h$ is the pullback to $Y$ of the hyperplane class in $\mathbf{P}^{2}$ and $e_{1}, \ldots, e_{9}$ are the exceptional curves in $Y$. We obtain the following:

Theorem 1.2. Let $Y$ be $\mathbf{P}^{2}$ blown up at 9 points; for fixed $g$ let $C_{n}$ be any class with $C_{n}^{2}=2 g+2 n-1$ and $C_{n} \cdot K=-1$. Let $N_{g}^{Y}\left(C_{n}\right)$ denote the number of genus $g$ curves in the class of $C_{n}$ passing through $g$ generic points. Then

$$
\sum_{n=0}^{\infty} N_{g}^{Y}\left(C_{n}\right) q^{C_{n}^{2} / 2}=\left(\sum_{k=1}^{\infty} k\left(\sum_{d \mid k} d\right) q^{k-1}\right)^{g} q^{-1 / 2} \prod_{m=1}^{\infty}\left(1-q^{m}\right)^{-12}=\frac{\left(D G_{2}\right)^{g}}{\sqrt{\Delta}} .
$$

Note that the Euler characteristic of $Y$ is 12 while the Euler characteristic of $K 3$ is 24 . For us the relevant manifestation of this fact is that elliptically fibered 
K3 surfaces have (generally) 24 nodal fibers while rational elliptic surfaces have (generally) 12 nodal fibers.

Since $N_{g}^{Y}\left(C_{n}\right)$ is an ordinary Gromov-Witten invariant (without family), it is an invariant for the deformation class of the symplectic structure on $Y$. In particular, $N_{g}^{Y}\left(C_{n}\right)$ is independent of the locations of those blow-up points in $\mathbf{P}^{2}$ and it is left invariant by Cremona transforms. For the genus zero case, the invariants were obtained by Göttsche and Pandharipande [20] where they computed the quantum cohomology for $\mathbf{P}^{2}$ blown up at an arbitrary number of points using the associativity law. Their numbers are in terms of two complicated recursive formulas and it is not obvious that the numbers that correspond to $N_{0}^{Y}\left(C_{n}\right)$ can be put together to form modular forms, but Theorem 1.2 can be verified term by term for $g=0$ using their recursion relations.

The foundations on which our calculations rest have been developed by $\mathrm{Li}$ and Tian ([26], 27], [28]) and also by Behrend-Fantechi and Siebert ([5], 6], 33], 34]). They construct the virtual fundamental cycle of the moduli space of stable maps both symplectically and algebraically and they show that the two constructions coincide in the projective case. Ionel and Parker have a different approach to computing $N_{0}^{Y}\left(C_{n}\right)$ that does not rely on [26].

Although our methods are completely different from those of Yau and Zaslow, for the sake of completeness we outline their beautiful argument for counting rational curves with $n$ nodes. Choose a smooth curve $C$ in the $K 3$ surface $X$ with $C \cdot C=$ $2 n-2$. By the adjunction formula, the genus of $C$ equals $n$. One can show that $C$ moves in a complete linear system of dimension $n$ using the Riemann-Roch theorem and a vanishing theorem. That is, $|C| \cong \mathbf{P}^{n}$.

Imposing a node will put one constraint on the linear system $|C|$. Therefore, by imposing $n$ nodes, one expects to see a finite number of rational curves with $n$ nodes. Define $N_{0}(n)$ to be this number. Now look at the compactified universal Jacobian $\pi: \overline{\mathcal{J}} \rightarrow|C|$ for this linear system (cf. Bershadsky, Sadov, and Vafa [8]). If one assumes that all the curves in $|C|$ are reduced and irreducible, then $\overline{\mathcal{J}}$ is a smooth hyperkähler manifold of dimension $2 n$.

If one assumes that each member in the linear system $|C|$ has at most nodal singularities, then one can argue that for any $C^{\prime} \in|C|$ the Euler characteristic of $\pi^{-1}\left(C^{\prime}\right)$ is always zero unless $C^{\prime}$ is a rational curve with $n$ nodes. In the latter case, the Euler characteristic of $\pi^{-1}\left(C^{\prime}\right)$ equals one. One concludes that $\chi(\overline{\mathcal{J}})=N_{0}(n)$.

On the other hand, $\overline{\mathcal{J}}$ is birational to the Hilbert scheme $\mathcal{H}_{n}$ of $n$ points in $X$, which is again another smooth hyperkähler manifold. Using a result of Batyrev [2] (cf. Huybrechts [23]) which states that compact, birationally equivalent, projective, Calabi-Yau manifolds have the same Betti numbers, one can conclude that $N_{0}(n)=$ $\chi\left(\mathcal{H}_{n}\right)$.

Then one uses the result of Göttsche [19], who used Deligne's answer to the Weil conjecture to compute (among other things) the Euler characteristic of $\mathcal{H}_{n}$ :

$$
\sum_{n=0}^{\infty} \chi\left(\mathcal{H}_{n}\right) q^{n}=\prod_{m=1}^{\infty}\left(1-q^{m}\right)^{-24}
$$

Finally, combining these results, one obtains

$$
F_{0}=\sum_{n=0}^{\infty} N_{0}(n) q^{n-1}=q^{-1} \prod_{m=1}^{\infty}\left(1-q^{m}\right)^{-24}=\Delta^{-1} .
$$


Our method is more direct than the Yau-Zaslow argument using symplectic geometry and Gromov-Witten invariants for families of symplectic structures, thus avoiding the characteristic $p$ methods employed by Göttsche and Batyrev. Our method also works in arbitrary genus.

We end this introduction with some speculation into the meaning of our results. Ordinary Gromov-Witten invariants give rise to the quantum cohomology ring. There may be a corresponding structure in the context of Gromov-Witten invariants for families. The computation of Theorem 1.1 shows that there is structure amongst these invariants and suggests that there should be an interesting theory of quantum cohomology that encodes it.

The ordinary quantum cohomology ring of $X$ gives a Frobenius structure on $H^{*}(X ; \mathbf{C})$ and the (generalized) mirror conjecture states that this Frobenius structure is equivalent to a Frobenius structure arising from some sort of "mirror object" (see [17] or [30] or [13]). In the case of a Calabi-Yau 3-fold, the mirror object is a family of Calabi-Yau 3-folds and the Frobenius structure arises from its variation of Hodge structure. Theorem 1.1] shows that the Gromov-Witten invariants for $K 3$ with its twistor family can be expressed in terms of quasi-modular forms. If there is a quantum cohomology theory associated to the Gromov-Witten invariants for families such as the twistor family and a corresponding mirror conjecture, then our theorem should provide clues as to what the "mirror object" of $K 3$ with its twistor family should be.

This paper is organized as follows. In section 2 we define invariants for families of symplectic structures; in section 3 we discuss twistor families and define $N_{g}(n)$; in section 4 we compute $N_{g}(n)$ and prove our main theorem; in section 5 we analyze the moduli spaces and compute local contributions; and in section [6 we apply similar techniques to $\mathbf{P}^{2}$ blown up at nine points. In Appendix $\mathrm{A}$ we prove some general results about virtual classes and in Appendix B we prove a result about infinitesimal deformations.

The authors are pleased to acknowledge helpful conversations with A. Bertram, A. Givental, L. Göttsche, T. Graber, E. Ionel, A. Liu, P. Lu, D. Maclagan, D. McKinnon, T. Parker, S. Schleimer, C. Taubes, A. Todorov, and S.-T. Yau. The authors especially thank L. Göttsche for sharing early versions of his conjecture with us and for providing many other valuable communications. We would like to thank R. Pandharipande and L. Göttsche for sending us their Maple program to verify our results. Additionally we thank the Park City Mathematics Institute for support and providing a stimulating environment where part of this work was carried out.

We also thank the referees for many valuable comments.

\section{INVARIANTS OF FAMILIES OF SYMPLECTIC STRUCTURES}

In this section, we introduce an invariant for a family of symplectic structures $\omega_{B}: B \rightarrow \Omega_{\text {sympl }}^{2}(X)$ on a compact manifold $X$. Here $B$ is an oriented, compact manifold and $\omega_{B}$ is a smooth map into the space of symplectic forms $\Omega_{\text {sympl }}^{2}(X)$. This invariant is a direct generalization of the Gromov-Witten invariants. Roughly speaking, it counts the number of maps $u: \Sigma \rightarrow X$ which are holomorphic with respect to some almost complex structure in a generic family compatible with $\omega_{B}$. Kronheimer [25] and $\mathrm{Li}$ and Liu [29] have also studied invariants for families of symplectic structures and obtained interesting results. 
In their paper on Gromov-Witten invariants for general symplectic manifolds, Li and Tian [28] set up a general framework for constructing invariants (this is also done by Siebert in [33]). Their results are easy to adapt to our setting but we remark that, in the case of interest, the full Li-Tian machinery is not needed and the techniques of Ruan-Tian [31 would suffice. This is because 2-dimension families of symplectic structures on a 4-manifold behave like the semi-positive case for the ordinary invariants, i.e. the (perturbed) moduli spaces are compactified by strata of codimension at least 2. The definition of Gromov-Witten invariants for families is also contained as part of the very general approach of Ruan [32]. We employ the Li-Tian machinery because they are also able to relate their symplectic constructions to their purely algebraic ones (27] and 26] ). In [34, Siebert relates his symplectic invariants to the algebraic invariants of Behrend-Fantechi [6].

Let $X$ be a compact smooth manifold. Suppose that $\omega_{B}$ is a smooth family of symplectic structures on $X$ parameterized by an oriented, compact manifold $B$. Let $J_{B}: B \rightarrow \mathcal{J}(X)$ be a smooth family of almost complex structures on $X$ such that $J_{t}=J_{B}(t)$ is compatible with $\omega_{t}=\omega_{B}(t)$ for any $t \in B$. In particular, $g_{t}=\omega_{t}\left(\cdot, J_{t} \cdot\right)$ is a family of Riemannian metrics on $X$. It is not difficult to see that $J_{B}$ always exists and is unique up to homotopy. This follows from the fact that the space of all almost complex structures compatible with a fixed symplectic form is contractible.

Given $X$ and $\omega_{B}$ as above, we shall define Gromov-Witten invariants for families as a homomorphism:

$$
\Psi_{(A, g, k)}^{\left(X, \omega_{B}\right)}: \bigotimes_{i=1}^{k} H^{a_{i}}(X, \mathbf{Q}) \otimes H^{b}\left(\overline{\mathcal{M}}_{g, k}, \mathbf{Q}\right) \rightarrow \mathbf{Q},
$$

with

$$
\sum_{i=1}^{k} a_{i}+b=2 c_{1}(X)(A)+2 k+\operatorname{dim} B+(\operatorname{dim} X-6)(1-g) .
$$

Here $A \in H_{2}(X, \mathbf{Z})$ and $\overline{\mathcal{M}}_{g, k}$ is the Deligne-Mumford compactification of the moduli space of Riemann surfaces of genus $g$ with $k$ distinct marked points (define $\overline{\mathcal{M}}_{g, k}$ to be a point if $2 g+k<3$ ).

For any particular symplectic structure $\omega_{t}$ and the corresponding almost complex structure $J_{t}, \mathrm{Li}$ and Tian define a section $\Phi_{t}$ of $E \rightarrow \overline{\mathcal{F}_{A}}(X, g, k)$ (we recall the definition below) which is equivalent to the Cauchy-Riemann operator. These sections depend on $t \in B$ smoothly so that we have a section $\Phi$ of $E \rightarrow \overline{\mathcal{F}}_{A}(X, g, k) \times B$.

Let us first recall their notations:

A stable map with $k$ marked points is a tuple $\left(f, \Sigma ; x_{1}, \ldots, x_{k}\right)$ satisfying:

(i) $\Sigma=\bigcup_{i=1}^{m} \Sigma_{i}$ is a connected normal crossing projective curve and the $x_{i}$ 's are distinct smooth points on $\Sigma$,

(ii) $f$ is continuous and $\left.f\right|_{\Sigma_{i}}$ can be lifted to a smooth map on the normalization of $\Sigma_{i}$, and

(iii) if $\Sigma_{i}$ is a smooth rational curve such that $f\left(\Sigma_{i}\right)$ represents a trivial homology class in $H_{2}(X, \mathbf{Q})$, then the cardinality of $\Sigma_{i} \cap\left(\left\{x_{1}, \ldots, x_{k}\right\} \cup S(\Sigma)\right)$ is at least three where $S(\Sigma)$ is the singular set of $\Sigma$.

Two stable maps $\left(f, \Sigma ; x_{1}, \ldots, x_{k}\right)$ and $\left(f^{\prime}, \Sigma ; x_{1}^{\prime}, \ldots, x_{k}^{\prime}\right)$ are equivalent if there is a biholomorphism $\sigma: \Sigma \rightarrow \Sigma^{\prime}$ such that $\sigma\left(x_{i}\right)=x_{i}^{\prime}$ for $1 \leq i \leq k$ and 
$f^{\prime}=f \circ \sigma$. We denote the space of equivalent classes of stable maps of genus $g$ with $k$ marked points and with total homology class $A$ by $\overline{\mathcal{F}}_{A}(X, g, k)$ and the subspace consisting of equivalent classes of stable maps with smooth domain by $\mathcal{F}_{A}(X, g, k)$. The topology of $\overline{\mathcal{F}}_{A}(X, g, k)$ can be defined by sequential convergence. Next they introduced a generalized bundle $E$ over $\overline{\mathcal{F}}_{A}(X, g, k)$ as follows: For any $\left[\left(f, \Sigma ; x_{1}, \ldots, x_{k}\right)\right] \in \overline{\mathcal{F}}_{A}(X, g, k)$, the fiber of $E$ consists of all $f^{*} T X$-valued $(0,1)$-forms over the normalization of $\Sigma$. Equipped with the continuous topology, $E$ is a generalized bundle over $\overline{\mathcal{F}}_{A}(X, g, k)$ in the sense of $\mathrm{Li}$ and Tian.

For each $t \in B$, there is a section of $E$ given by the Cauchy-Riemann operator defined by $J_{t}$ : Namely, for any $\left[\left(f, \Sigma ; x_{1}, \ldots, x_{k}\right)\right] \in \overline{\mathcal{F}}_{A}(X, g, k)$, we have $\Phi_{t}\left(f, \Sigma ; x_{1}, \ldots, x_{k}\right)=d f+J_{t} \circ d f \circ j_{\Sigma}$ where $j_{\Sigma}$ is the complex structure of $\Sigma$. Putting different $t \in B$ together, we have a section $\Phi$ of $E$ over $\overline{\mathcal{F}}_{A}(X, g, k) \times B$ given by

$$
\Phi\left(\left[\left(f, \Sigma ; x_{1}, \ldots, x_{k}\right)\right], t\right)=d f+J_{t} \circ d f \circ j_{\Sigma} .
$$

The following theorems are easy adaptations of those in of Li and Tian found in 28]:

Theorem 2.1. The section $\Phi$ gives rise to a generalized Fredholm orbifold bundle with the natural orientation and of index

$$
2 c_{1}(X)[A]+2 k+\operatorname{dim} B+(\operatorname{dim} X-6)(1-g) .
$$

Theorem 2.2. Let $\omega_{B}$ and $\omega_{B}^{\prime}$ be two families of symplectic structures on $X p a-$ rameterized by $B$. Suppose that they are equivalent to each other under deformations for families. Let $J_{B}$ and $J_{B}^{\prime}$ be two families of almost complex structures on $X$ compatible with corresponding symplectic structures.

Suppose that $\Phi$ and $\Phi^{\prime}$ are the corresponding section of $E$ over $\overline{\mathcal{F}}_{A}(X, g, k) \times$ B. Then $\Phi$ and $\Phi^{\prime}$ are homotopic to each other as generalized Fredholm orbifold bundles.

Using the main theorem of $\mathrm{Li}$ and Tian in their paper, there is an Euler class $e\left(\left[\Phi: \overline{\mathcal{F}}_{A}(X, g, k) \times B \rightarrow E\right]\right)$ in $H_{r}\left(\overline{\mathcal{F}_{A}}(X, g, k) \times B, \mathbf{Q}\right)$ with $r=2 c_{1}(X)[A]+$ $2 k+\operatorname{dim} B+(\operatorname{dim} X-6)(1-g)$. This class is called the virtual fundamental cycle of the moduli space of holomorphic stable maps $\overline{\mathcal{M}}_{g, k}(X, B ; A)$. We denote it by $\left[\overline{\mathcal{M}}_{g, k}(X, B ; A)\right]^{\text {vir }}$.

To define the invariant for $\omega_{B}$, we consider the following two maps. First we have the evaluation map $e: \overline{\mathcal{F}}_{A}(X, g, k) \times B \rightarrow X^{k}$ :

$$
e\left(\left(f, \Sigma ; x_{1}, \ldots, x_{k}\right), t\right)=\left(f\left(x_{1}\right), \ldots, f\left(x_{k}\right)\right),
$$

and second we have the forgetful map $\pi_{g, k}: \overline{\mathcal{F}}_{A}(X, g, k) \times B \rightarrow \overline{\mathcal{M}}_{g, k}$ :

$$
\pi_{g, k}\left(\left(f, \Sigma ; x_{1}, \ldots, x_{k}\right), t\right)=\operatorname{red}\left(\Sigma ; x_{1}, \ldots, x_{k}\right) .
$$

Here $\operatorname{red}\left(\Sigma ; x_{1}, \ldots, x_{k}\right)$ is the stable reduction of $\left(\Sigma ; x_{1}, \ldots, x_{k}\right)$ that is obtained by contracting all of its non-stable irreducible components.

Now we can define the invariants

$$
\Psi_{(A, g, k)}^{(X, B)}: H^{*}(X, \mathbf{Q})^{\otimes k} \otimes H^{*}\left(\overline{\mathcal{M}}_{g, k}, \mathbf{Q}\right) \rightarrow \mathbf{Q}
$$

by

$$
\Psi_{(A, g, k)}^{(X, B)}\left(\alpha_{1}, \ldots, \alpha_{k} ; \beta\right)=\left(e^{*}\left(\alpha_{1} \otimes \cdots \otimes \alpha_{k}\right) \cup \pi_{g, k}^{*}(\beta)\right)\left[\overline{\mathcal{M}}_{g, k}(X, B ; A)\right]^{v i r}
$$


for any $\alpha_{1}, \ldots, \alpha_{k} \in H^{*}(X, \mathbf{Q})$ and $\beta \in H^{*}\left(\overline{\mathcal{M}}_{g, k}, \mathbf{Q}\right)$. We drop $\beta$ from the notation when $\beta=1$.

Theorem 2.3. $\Psi_{(A, g, k)}^{(X, B)}$ is an invariant of the deformation class of the family of symplectic structures $\omega_{B}$.

If $\left(\hat{\alpha}_{1}, \ldots, \hat{\alpha}_{k}\right)$ are geometric cycles in $X$ that are Poincaré dual to $\left(\alpha_{1}, \ldots, \alpha_{k}\right)$ and $\hat{\beta}$ is a cycle in $\overline{\mathcal{M}}_{g, k}$ dual to $\beta$, then $\Psi_{(A, g, k)}^{(X, B)}\left(\alpha_{1}, \ldots, \alpha_{k} ; \beta\right)$ counts the number of stable maps $f: \Sigma_{g} \rightarrow X$ so that

1. $f\left(\Sigma_{g}\right)$ represents the class $A$,

2. $f$ is $J_{t}$-holomorphic for some $t \in B$,

3. $f\left(x_{i}\right)$ lies on $\hat{\alpha}_{i}$, and

4. the stable reduction of $\Sigma_{g}$ lies in $\hat{\beta} \subset \overline{\mathcal{M}}_{g, k}$.

One is usually interested in $\hat{\beta}=\overline{\mathcal{M}}_{g, k}$ (i.e. $\beta=1$ ), or sometimes $\hat{\beta}=$ pt. $\in \overline{\mathcal{M}}_{g, k}$.

\section{Twistor families of $K 3$ surfaces and the Definition of $N_{g}(n)$}

In this section we collect some general facts about $K 3$ surfaces and their twistor families. We show that every twistor family is deformation equivalent and we define $N_{g}(n)$ in terms of the Gromov-Witten invariant for this family. We show that when $X$ is projective and $|C|$ has only reduced and irreducible curves, $N_{g}(n)$ coincides with the enumerative count defined by algebraic geometers (see [15]). The results of the section are summarized in Definition 3.4 .

A $K 3$ surface is a simply-connected, compact, complex surface $X$ with $c_{1}(X)=0$. For a general reference on $K 3$ surfaces and twistor families we refer the reader to [1] or 9. Any pair of $K 3$ surfaces is deformation equivalent and hence diffeomorphic. A marking of a $K 3$ surface $X$ is an identification of the intersection pairing $\left(H^{2}(X ; \mathbf{Z}), Q_{X}\right)$ with the fixed unimodular form $Q=-2 E_{8} \oplus 3\left(\begin{array}{ll}0 & 1 \\ 1 & 0\end{array}\right)$. The space of marked $K 3$ surfaces forms a connected, 20 complex dimensional moduli space.

The complex structure on a marked $K 3$ surface $X$ is determined by how the line $H^{0,2}(X)$ sits in $Q \otimes \mathbf{C}$. To make this precise, define the period $\Omega_{X}$ of $X$ to be the element of the period domain

$$
\mathcal{D}=\{\Omega \in \mathbf{P}(Q \otimes \mathbf{C}):\langle\Omega, \bar{\Omega}\rangle=0,\langle\Omega, \Omega\rangle>0\}
$$

given by the image of $H^{0,2}(X)$ under the marking.

The Torelli theorem states that $\mathcal{D}$ is the moduli space of marked $K 3$ surfaces, i.e. every marked $K 3$ surface corresponds uniquely to its period point in $\mathcal{D}$ and every $\Omega \in \mathcal{D}$ is the period point of some $K 3$ surface.

For a fixed $K 3$ surface $X$ with period $\Omega_{X}$, a class $\omega_{X} \in Q \otimes \mathbf{C}$ is a Kähler class for $\left(X, \Omega_{X}\right)$ if and only if $\left\langle\omega_{X}, \Omega_{X}\right\rangle=0,\left\langle\omega_{X}, \bar{\Omega}_{X}\right\rangle=0, \omega_{X}=\bar{\omega}_{X}$, and $\left\langle\omega_{X}, \omega_{X}\right\rangle>0$. For any Kähler $K 3$ surface $\left(X, \Omega_{X}, \omega_{X}\right)$ there is a unique hyperkähler metric by Yau's proof of the Calabi conjecture [36]. A hyperkähler metric $g$ determines a 2sphere worth of Kähler structures, namely the unit sphere in the space $\mathcal{H}_{+, g}^{2}$ of selfdual harmonic forms. We can describe the corresponding 2-sphere of period points as follows. Consider the projective plane spanned by $\left\langle\Omega_{X}, \bar{\Omega}_{X}, \omega_{X}\right\rangle$ in $\mathbf{P}(Q \otimes \mathbf{C})$. Since $\left\langle\Omega_{X}, \bar{\Omega}_{X}, \omega_{X}\right\rangle$ spans $\mathcal{H}_{+, g}^{2} \otimes \mathbf{C}$, the intersection of this projective plane and the period domain is the quadric determined by $\langle\Omega, \bar{\Omega}\rangle=0$. This is a smooth plane quadric and hence a 2 -sphere. This 2 -sphere of complex structures together with the corresponding 2-sphere of Kähler structures we call a twistor family. We will 
use the notations $\left(J_{T}, \omega_{T}\right)$ to refer to a twistor family and $J_{t}, \omega_{t}$ for $t \in T$ to refer to individual members.

The following proposition was explained to us by Andrei Todorov:

Proposition 3.1. Let $\left(X, \Omega_{X}, \omega_{X}\right)$ be a marked, Kähler $K 3$ surface and $\left(J_{T}, \omega_{T}\right)$ the corresponding twistor family. Let $C \in H^{2}(X ; \mathbf{Z})$ be a class of square $C^{2} \geq-2$. Then there is exactly one member $t \in T$ for which there is a $J_{t}$-holomorphic curve in the class of $C$.

Proof. A class $C$ of square -2 or larger admits a holomorphic curve if and only if $C \in H^{1,1}(X ; \mathbf{Z})$ and $C$ pairs positively with the Kähler class. Since $C$ is a real class, $C \in H^{1,1}$ if and only if $\left\langle C, \Omega_{X}\right\rangle=0$. This equation determines a hyperplane in $\mathbf{P}\left(H_{+}^{2}\right)$ and so meets the twistor space in 2 points $\pm \Omega_{0}$ (since the twistor space is a quadric). Then exactly one of $\pm \Omega_{0}$ will have its corresponding Kähler class pair positively with $C$.

We next show that every twistor family is the same up to deformation.

Proposition 3.2. Let $X_{1}$ and $X_{2}$ be two Kähler $K 3$ surfaces. Then the corresponding twistor families $T_{0}$ and $T_{1}$ are deformation equivalent.

Proof. The moduli space of $K 3$ surfaces is connected and the space of hyperkähler structures for a fixed $K 3$ surface is contractible (it is the Kähler cone). Therefore, the space parameterizing hyperkähler $K 3$ surfaces $\left(X, \Omega_{X}, \omega_{X}\right)$ is also connected. We can thus find a path $\left(X_{s}, \Omega_{s}, \omega_{s}\right), s \in[0,1]$, connecting $X_{0}$ to $X_{1}$ where the twistor family of $\omega_{i}$ is $T_{i}$ for $i=0,1$. By then associating to each hyperkähler structure $\omega_{s}$ its twistor family $T_{s}$, we obtain a continuous deformation of $T_{0}$ to $T_{1}$.

From this proposition we see that the Gromov-Witten invariants for a twistor family are independent of the choice of a twistor family. We can thus write unambiguously

$$
\Psi_{(C, g, k)}^{(K 3, T)}: H^{*}(K 3 ; \mathbf{Z})^{\otimes k} \otimes H^{*}\left(\overline{\mathcal{M}}_{g, k}\right) \rightarrow \mathbf{Q} .
$$

We are primarily interested in the invariants that count stable maps without fixing the complex structure on the domain. That is, the invariants obtained using the Poincaré dual of the fundamental class of $\overline{\mathcal{M}}_{g, k}$ (i.e. $\beta=1$ ). It is enough to consider those constraints that come from the generator of $H^{4}(K 3, \mathbf{Z})$; these count curves passing through fixed generic points. The invariants with the constraint that the $k$ th point lies on a fixed generic cycle dual to an element $\beta \in H^{2}(K 3)$ can be computed in terms of the invariants for $k-1$ constraints and the pairing $\beta \cdot C$. For this reason, constraining the invariants by elements of $H^{2}(K 3)$ is uninteresting, and of course elements of $H^{0}(K 3)$ provide no constraints at all.

Thus the only possible invariant of interest is $\Psi_{(C, g, g)}^{(K 3, T)}\left(\left[p_{1}\right]^{\vee}, \ldots,\left[p_{g}\right]^{\vee}\right)$ where $p_{1}, \ldots, p_{g}$ are points in $X$ and we use $(\cdot)^{\vee}$ to denote the Poincáre dual of a homology class.

An important observation about the twistor family is the following.

Proposition 3.3. If $f: K 3 \rightarrow K 3$ is an orientation preserving diffeomorphism, then the pullback family $f^{*}\left(\omega_{T}\right)$ is deformation equivalent to $\omega_{T}$; thus

$$
\Psi_{C, g, g}^{(K 3, T)}\left(\left[p_{1}\right]^{\vee}, \ldots,\left[p_{g}\right]^{\vee}\right)=\Psi_{f_{*}(C), g, g}^{(K 3, T)}\left(\left[p_{1}\right]^{\vee}, \ldots,\left[p_{g}\right]^{\vee}\right) .
$$


Proof. Let $\omega_{T}$ be the twistor family associated to a hyperkähler metric $g$. Then $f^{*}\left(\omega_{T}\right)$ is the twistor family associated to the hyperkähler metric $f^{*}(g)$ and so by Proposition 3.2 they are deformation equivalent. Of course we also have that $f^{*}\left(\left[p_{i}\right]^{\vee}\right)=\left[p_{i}\right]^{\vee}$, hence the proposition.

The $K 3$ surface has a big diffeomorphism group in the sense of Friedman and Morgan [16], which means that every automorphism of the lattice $Q_{X}$ which preserves spinor norm can be realized by an orientation preserving diffeomorphism. In particular, one can take any primitive class $C \in H_{2}(K 3 ; \mathbf{Z})$ to any other primitive class with the same square via an orientation preserving diffeomorphism.

We are now in a position to define $N_{g}(n)$. By the adjunction formula, a holomorphic curve of genus $g$ with $n$ nodes will be in a class $C$ with square $C^{2}=2(g+n)-2$.

Definition 3.4. Let $C$ be any primitive class with $C^{2}=2(g+n)-2$. We define the number $N_{g}(n)$ by

$$
N_{g}(n)=\Psi_{(C, g, g)}^{(K 3, T)}\left(\left[p_{1}\right]^{\vee}, \ldots,\left[p_{g}\right]^{\vee}\right) .
$$

By Proposition 3.3, $N_{g}(n)$ is independent of the choice of the primitive class $C$. By Proposition 3.2, $N_{g}(n)$ is independent of the choice of twistor family. Finally, in the case of a projective $K 3$ surface with an effective divisor in the class of $C$, Proposition 3.1 shows that $N_{g}(n)$ counts holomorphic maps $f: D \rightarrow X$ of genus $g$ curves to $X$ with image in $|C|$ and passing through $g$ generic points.

Because the invariants $N_{g}(n)$ count maps of genus $g$ curves, they are a priori different than the actual count of (geometric) genus $g$ curves. In general, GromovWitten type invariants may also count maps that collapse components of positive genus to a point or multiply-covered components. Even if there are no multiplycovered or collapsed components, one should assign multiplicities to curves with singularities more complicated than nodes. A consistent way of doing this is constructed in 15. where they show that the multiplicities are positive, integral, and coincide with the length of the (zero-dimensional) moduli space of genus $g$ stable maps to the curve.

If a Gromov-Witten type invariant counts only curves of geometric genus $g$, possibly with positive, integral multiplicities for curves with singularities worse than nodes, we will say that the invariant is weakly enumerative. If in addition, all the curves are nodal so that each curve is counted exactly once, we say the invariant is strongly enumerative or just enumerative 2

Theorem 3.5. If $X$ is generic among those K3 surfaces admitting a curve in the class $[C]$, and the $g$ points are chosen generically, then the invariant $N_{g}(n)$ is strongly enumerative, that is, $N_{g}(n)$ is precisely the number of geometric genus $g$ curves in $|C|$ passing through g generic points.

Proof. 3 The assumption that the $K 3$ surface $X$ is generic among those admitting a curve in the class of $C$ guarantees that the primitive class $C$ generates the Picard group. Suppose that the invariant $N_{g}(n)$ differs from the actual count of curves $\Sigma \in|C|$ of genus $g$ passing through $g$ general points (curves possibly counted with

\footnotetext{
${ }^{2}$ For example, in 20] Göttsche and Pandharipande show that the genus 0 Gromov-Witten invariants of $\mathbf{P}^{2}$ blown up at $N$ points is strongly enumerative for $N<10$ and their arguments show additionally that the invariants are weakly enumerative for all $N$.

${ }^{3}$ This argument is due to Lothar Göttsche. We are grateful to him for showing it to us.
} 
multiplicities). Then there is an extra map $D \rightarrow X$ of a curve of arithmetic genus $g$ that has some contracted components and the rest of the map is generically injective with irreducible image. Let $C_{1}$ be a contracted component. Since the $g$ marked points have to go to $g$ distinct points on $X, C_{1}$ can have at most 1 marked point. By stability then, either the geometric genus $g\left(C_{1}\right)$ is larger than 0 or $C_{1}$ intersects the rest of $D$ in at least 2 points. Since the image of $D$ is irreducible, the contracted components cannot all be genus 0 unless the dual graph of $D$ is not a tree. Thus either $D$ has a contracted component of genus greater than 0 or the dual graph of $D$ is not a tree. In either case, the geometric genus of the image is smaller than the arithmetic genus of $D$ and thus the image is a curve of genus less than $g$ passing through $g$ points. This does not occur for $g$ generic points by a dimension count.

This shows that $N_{g}(n)$ is weakly enumerative; to get the strongly enumerative result, we evoke the very recent proof of Chen that for a generic algebraic $K 3$ surface with a primitive ample class $[C]$, all the rational curves in $|C|$ are nodal 11]. A simple corollary of Chen's result is that the generic genus $g$ member of $|C|$ is also nodal [12]. Thus under this genericity assumption, $N_{g}(n)$ always counts nodal curves, hence with multiplicity 1.

Remark 3.6. The conjectured formula of Yau and Zaslow applies to non-primitive classes as well. The above definition could be made for arbitrary classes $C$, but $a$ priori $N_{g}(n)$ would also depend on the divisibility of $C$. Our method of computing $N_{g}(n)$ only applies to primitive classes, so the Yau-Zaslow conjecture remains open for the non-primitive classes.

\section{Computation of $N_{g}(n)$}

To compute $N_{g}(n)$ we are free to choose any family of symplectic structures deformation equivalent to the twistor family and any primitive class $C$ with $C^{2}=$ $2(g+n)-2$. Let $X$ be an elliptically fibered $K 3$ surface with a section and 24 nodal singular fibers $N_{1}, \ldots, N_{24}$. Endow $X$ with a hyperkähler metric and let $\left(\omega_{T}, J_{T}\right)$ be the corresponding twistor family. Let $S$ denote the section and $F$ the class of the fiber so that $F^{2}=0, F \cdot S=1$, and $S^{2}=-2$. Let $C$ be the class $S+(n+g) F$ and fix $g$ generic points $p_{1}, \ldots, p_{g}$ not on $S$ that lie on $g$ distinct smooth fibers which we label $F_{1}, \ldots, F_{g}$. We denote the intersections of $N_{i}$ and $F_{j}$ with $S$ by $y_{i}$ and $z_{j}$ respectively (see Figure 1 ).

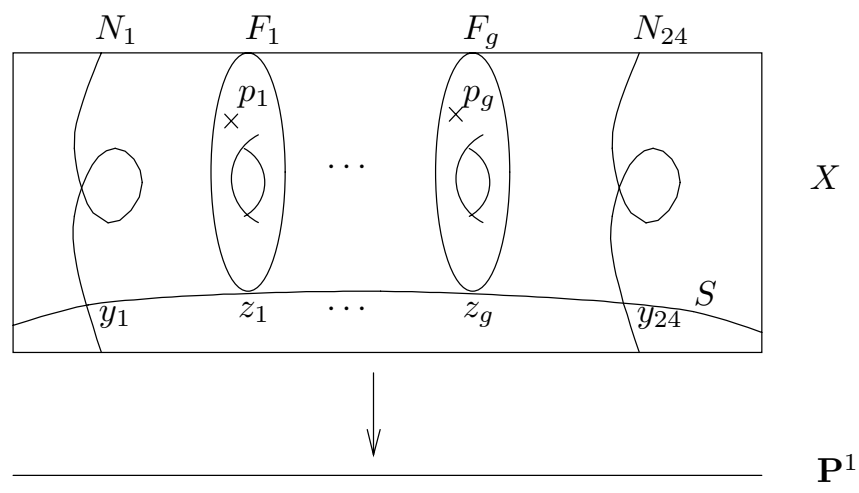

FIGURE 1. 
Recall from Definition 3.4 that

$$
N_{g}(n)=\Psi_{C, g, g}^{(K 3, T)}\left(\left[p_{1}\right]^{\vee}, \ldots,\left[p_{g}\right]^{\vee}\right)
$$

This is given as the evaluation of the class $e^{*}\left(\left[p_{1}\right]^{\vee} \otimes \cdots \otimes\left[p_{g}\right]^{\vee}\right)$ on the cycle $\left[\overline{\mathcal{M}}_{g, g}(X, T, C)\right]^{\text {vir }}$. We abbreviate $\overline{\mathcal{M}}_{g, g}(X, T, C)$ by just $\overline{\mathcal{M}}_{g, g}(X)$ and we let $\overline{\mathcal{M}}(X, \mathbf{p}) \subset \overline{\mathcal{M}}_{g, g}(X)$ denote the subspace of maps which send the $j$ th marked point to $p_{j}$. The invariant $N_{g}(n)$ is also given directly as a 0 -dimensional class on $\overline{\mathcal{M}}_{g, g}(X, \mathbf{p})$ (see Appendix $\mathbf{A}$ ).

By Proposition [3.1, there is a unique $t_{0} \in T$ so that there are $J_{t_{0}}$-holomorphic curves in the class of $C$. This $J_{t_{0}}$ must be the original elliptically fibered complex structure. Thus $\overline{\mathcal{M}}(X, \mathbf{p})$ consists of stable holomorphic maps whose images are in the linear system $|S+(n+g) F|$ and contain the points $p_{1}, \ldots, p_{g}$. Because of the elliptic fibration, the linear system $|C|$ is easy to analyze.

The dimension of $|S+(n+g) F|$ is $n+g$ and consists solely of reducible curves which are each a union of the section and $(n+g)$ (not necessarily distinct) fibers. Since the image contains the points $p_{1}, \ldots, p_{g}$, it contains the corresponding smooth fibers $F_{1}, \ldots, F_{g}$. The image of a map in $\overline{\mathcal{M}}(X, \mathbf{p})$ must therefore be the union of the section $S$, the $g$ fibers $F_{1}, \ldots, F_{g}$, and some number of nodal fibers (possibly counted with multiplicity). We summarize this discussion in the following

Proposition 4.1. Let $\overline{\mathcal{M}}(X, \mathbf{p})$ be the moduli space of genus $g$, g-marked, stable maps in the class of $C=S+(g+n) F$ satisfying $f\left(x_{j}\right)=p_{j}$ where $x_{j}$ is the jth marked point. Let $\pi: \overline{\mathcal{M}}(X, \mathbf{p}) \rightarrow \mathbf{P}\left(H^{0}(X, C)\right)$ be the natural projection onto the linear system $|C|$. Then $\operatorname{Im}(\pi)$ is a finite number of points labeled by the vectors $\mathbf{a}=\left(a_{1}, \ldots, a_{24}\right)$ and $\mathbf{b}=\left(b_{1}, \ldots, b_{g}\right)$ where $a_{i} \geq 0, b_{j} \geq 1$, and $\sum a_{i}+\sum b_{j}=n+g$. The corresponding divisor in $|C|$ is

$$
S+\sum_{j=1}^{g} b_{j} F_{j}+\sum_{i=1}^{24} a_{i} N_{i}
$$

where $F_{j}$ is the smooth fiber containing $p_{j}$ and $N_{1}, \ldots, N_{24}$ are the nodal fibers.

The proposition implies that $\overline{\mathcal{M}}(X, \mathbf{p})$ is the disjoint union of components $\overline{\mathcal{M}}_{\mathbf{a}, \mathbf{b}}$ labeled by the vectors $\mathbf{a}$ and $\mathbf{b}$. In section $\mathbf{5}$ we analyze the moduli spaces $\overline{\mathcal{M}}_{\mathbf{a}, \mathbf{b}}$ in detail. The main result of that section (Theorem [5.10) is that the contribution to $N_{g}(n)$ from $\overline{\mathcal{M}}_{\mathbf{a}, \mathbf{b}}$ is the product of the local contributions:

$$
\prod_{j=1}^{g} b_{j} \sigma\left(b_{j}\right) \prod_{i=1}^{24} p\left(a_{i}\right)
$$

Our main theorem follows from this and some manipulations with the generating functions. Recall that the generating function of the partition function $p(l)$ is $\prod_{m=1}^{\infty}\left(1-q^{m}\right)^{-1}$. Recall also that $\mathbf{a}$ is a 24 -tuple of integers with $a_{i} \geq 0$, $\mathbf{b}$ is a $g$-tuple of integers with $b_{j} \geq 1$, and $|\mathbf{a}|+|\mathbf{b}|=\sum_{i=1}^{24} a_{i}+\sum_{j=1}^{g} b_{j}=n+g$. We 
compute:

$$
\begin{aligned}
N_{g}(n) q^{n+g-1} & =\left(\sum_{\substack{\mathbf{a}, \mathbf{b} \\
|\mathbf{a}|+|\mathbf{b}|=n+g}} \prod_{j=1}^{g} b_{j} \sigma\left(b_{j}\right) \prod_{i=1}^{24} p\left(a_{i}\right)\right) q^{n+g-1} \\
& =q^{-1} \sum_{k=0}^{n} \sum_{\substack{|\mathbf{a}|=n-k \\
|\mathbf{b}|=g+k}} \prod_{j=1}^{g} b_{j} \sigma\left(b_{j}\right) q^{b_{j}} \prod_{i=1}^{24} p\left(a_{i}\right) q^{a_{i}}
\end{aligned}
$$

Summing over $n$ :

$$
\begin{aligned}
\sum_{n=0}^{\infty} N_{g}(n) q^{n+g-1} & =q^{-1} \sum_{n=0}^{\infty} \sum_{k=0}^{n}\left(\sum_{|\mathbf{b}|=g+k} \prod_{j=1}^{g} b_{j} \sigma\left(b_{j}\right) q^{b_{j}}\right)\left(\sum_{|\mathbf{a}|=n-k} \prod_{i=1}^{24} p\left(a_{i}\right) q^{a_{i}}\right) \\
& =q^{-1}\left(\sum_{|\mathbf{b}| \geq g} \prod_{j=1}^{g} b_{j} \sigma\left(b_{j}\right) q^{b_{j}}\right)\left(\sum_{|\mathbf{a}| \geq 0} \prod_{i=1}^{24} p\left(a_{i}\right) q^{a_{i}}\right) \\
& =q^{-1} \prod_{j=1}^{g}\left(\sum_{b_{j}=1}^{\infty} b_{j} \sigma\left(b_{j}\right) q^{b_{j}}\right) \prod_{i=1}^{24}\left(\sum_{a_{i}=0}^{\infty} p\left(a_{i}\right) q^{a_{i}}\right) \\
& =q^{-1}\left(\sum_{b=1}^{\infty} b \sigma(b) q^{b}\right)^{g} \prod_{m=1}^{\infty}\left(1-q^{m}\right)^{-24} \\
& =\frac{\left(D G_{2}\right)^{g}}{\Delta} .
\end{aligned}
$$

This proves our main theorem.

\section{AnAlysis OF MODUli SPACES AND LOCAL CONTRIBUtions}

The main goal of this section is to compute the contribution of the component $\overline{\mathcal{M}}_{\mathbf{a}, \mathbf{b}}$ to the invariant $N_{g}(n)$. Our strategy is simple in essence. We show that the moduli space can be written as a product of various other moduli spaces and that the obstruction theory splits into factors that pull back from obstruction theories on the other moduli spaces. We then show that those individual moduli-obstruction problems have many components, each of which can be identified with moduliobstruction problems arising for the Gromov-Witten invariants of $\mathbf{P}^{2}$ blown up multiple times. These contributions can then be determined by elementary properties of the Gromov-Witten invariants on blow ups of $\mathbf{P}^{2}$. Using Cremona transformations, these contributions can be shown to all either vanish or be equivalent to the number of straight lines between two points (one). The formula then follows from straightforward combinatorics.

This section is somewhat notationally heavy so to help the reader navigate we summarize the notation used. We use $\overline{\mathcal{M}}_{g, g}(X)$ for the full moduli space of stable maps of $g$-marked, genus $g$ curves to $X$ in the class of $C ; \overline{\mathcal{M}}(X, \mathbf{p})$ denotes the subspace of $\overline{\mathcal{M}}_{g, g}(X)$ where $x_{j}$, the $i$ th marked point, maps to $p_{j} . \overline{\mathcal{M}}(X, \mathbf{p})$ breaks into components $\overline{\mathcal{M}}_{\mathbf{a}, \mathbf{b}}$ indexed by vectors $\mathbf{a}=\left(a_{1}, \ldots, a_{24}\right)$ and $\mathbf{b}=\left(b_{1}, \ldots, b_{g}\right)$ determining the image of the map. We will use the index $i$ for things associated to the nodal fibers, e.g. $a_{i}, N_{i}$, and $D_{i}$, and we will use the index $j$ for those associated 
to the smooth fibers containing $p_{j}$, e.g. $b_{j}, F_{j}$, and $G_{j}$. The components $\overline{\mathcal{M}}_{\mathbf{a}, \mathbf{b}}$ break into further components $\overline{\mathcal{M}}_{s, \Lambda}$ indexed by "data" $(s(\mathbf{a}), \Lambda(\mathbf{b}))$ (Theorem 5.1). To prove Theorem 5.1 it is convenient to introduce $\overline{\mathcal{M}}_{a}$ which denotes the moduli space of genus 0 stable maps to $X$ with image $S+a N$ where $N$ is any fixed nodal fiber. The moduli space $\overline{\mathcal{M}}_{a}$ breaks into components because of the possibility of "jumping" behavior at the node of $N$. This behavior is encoded by certain kinds of sequences $s(a)=\left\{s_{n}(a)\right\}$ (we call admissible) and hence the components of $\overline{\mathcal{M}}_{a}$ are indexed by such sequences. We denote those components by $\overline{\mathcal{M}}_{s(a)}$. We compute the contribution of $\overline{\mathcal{M}}_{s(a)}$ by "matching" its virtual class with the virtual class on a moduli space of stable maps to a blow up of $\mathbf{P}^{2}$. This blow up is denoted $\tilde{P}$ and the relevant moduli space is denoted $\overline{\mathcal{M}}_{s(a)}^{\tilde{P}}$. Ultimately, we show that the contribution of each component to the invariant is either 0 or 1 ; those components that contribute 1 are those for which the relevant admissible sequences have a special property (we call such sequences 1-admissible). The contribution of $\overline{\mathcal{M}}_{\mathbf{a}, \mathbf{b}}$ is then obtained by counting how many possibilities there are for the data $(\Lambda(\mathbf{b}), s(\mathbf{a}))$ that have only 1-admissible sequences.

In subsection 5.1 we identify the components $\overline{\mathcal{M}}_{s(\mathbf{a}), \Lambda(\mathbf{b})}$ of $\overline{\mathcal{M}}_{\mathbf{a}, \mathbf{b}}$ and show that they are a product of spaces $\prod_{i} \overline{\mathcal{M}}_{s\left(a_{i}\right)}$. In subsection 5.2 we deal with the technical issue of showing that the virtual class of $\overline{\mathcal{M}}_{s, \Lambda}$ splits as a product of virtual classes defined on the factors. In subsection 5.3 we compute the virtual class of $\overline{\mathcal{M}}_{s\left(a_{i}\right)}$ by our matching technique.

5.1. Components of $\overline{\mathcal{M}}_{\mathbf{a}, \mathbf{b}}$. We begin by identifying the connected components of $\overline{\mathcal{M}}_{\mathbf{a}, \mathbf{b}}$. Call a sequence $\left\{s_{n}\right\}$ admissible if each $s_{n}$ is a positive integer and the index $n$ runs from some non-positive integer through some non-negative integer (the sequence could consist solely of $\left\{s_{0}\right\}$ for example). Write $|s|$ for $\sum_{n} s_{n}$.

Theorem 5.1. The connected components of $\overline{\mathcal{M}}_{\mathbf{a}, \mathbf{b}}$ are indexed by data $(\Lambda(\mathbf{b}), s(\mathbf{a}))$. The data $s(\mathbf{a})$ assigns for each $a_{i} \in \mathbf{a}$ an admissible sequence $\left\{s_{n}\left(a_{i}\right)\right\}$ such that $\left|s\left(a_{i}\right)\right|=a_{i}$. The data $\Lambda(\mathbf{b})$ assigns for each $b_{j} \in \mathbf{b}$ a sublattice of $\mathbf{Z} \oplus \mathbf{Z}$ of index $b_{j}$ and an element of the set $\left\{1,2, \ldots, b_{j}\right\}$. We write

$$
\overline{\mathcal{M}}_{\mathbf{a}, \mathbf{b}}=\coprod_{(s(\mathbf{a}), \Lambda(\mathbf{b}))} \overline{\mathcal{M}}_{(s, \Lambda)} .
$$

Remark 5.2. The number of sublattices of $\mathbf{Z} \oplus \mathbf{Z}$ of index $b$ is classically known and is given by $\sigma(b)=\sum_{d \mid b} d$. Thus we see that the number of possible choices of the data $\Lambda(\mathbf{b})$ is $\prod_{j=1}^{g} b_{j} \sigma\left(b_{j}\right)$.

Let $(f: C \rightarrow X) \in \overline{\mathcal{M}}_{\mathbf{a}, \mathbf{b}}$. Since the image of $f: C \rightarrow X$ is $S+\sum_{j} b_{j} F_{j}+$ $\sum_{i} a_{i} N_{i}$ and is reducible, $C$ must be reducible and its components must group into the set of components mapping to $S, F_{1}, \ldots, F_{g}$, and $N_{1}, \ldots, N_{24}$. Since the components mapping to each $F_{j}$ must have geometric genus at least 1 and the total geometric genus of $C$ is $g$, the components of $C$ mapping to $F_{j}$ must each be genus 1 and all other components of $C$ are rational. Furthermore, the dual graph of $C$ is a tree and the $g$ marked points are on the $g$ elliptic components which we call $G_{1}, \ldots, G_{g}$. Then since $f$ is a stable map, the image of all collapsed components

\footnotetext{
${ }^{4}$ Notation: from here on out we use $C$ to refer to the domain of a stable map instead of the homology class $C \in H_{2}(X)$ that we have previously used. Since we will not need to refer to the homology class much, this should not pose too much confusion.
} 
of $C$ must lie in the nodal fibers $N_{1}, \ldots, N_{24}$. We denote the component of $C$ mapping isomorphically onto $S$ also by $S$.

So far then, we can describe the domain $C$ as a rational curve $S$ that has attached to it $g$ marked elliptic curves $G_{1}, \ldots, G_{g}$ and 24 components $D_{1}, \ldots, D_{24}$ that are either empty (if $a_{i}=0$ ) or a tree of rational components. Furthermore, $\left.f\right|_{G_{j}}: G_{j} \rightarrow$ $F_{j}$ is a degree $b_{j}$ map preserving the intersection with $S$ and sending the marked point to $p_{j}$ and $f \mid D_{i}: D_{i} \rightarrow N_{i}$ has total degree $a_{i}$.

Using the intersection with $S$ as an origin for $G_{j}$ and $F_{j}$, we can identify the number of distinct possibilities for the map $f: G_{j} \rightarrow F_{j}$ with the number of degree $b_{j}$ homomorphisms onto a fixed elliptic curve $F_{j}$. This is precisely the number of index $b_{j}$ sublattices of $\mathbf{Z} \oplus \mathbf{Z}$. Additionally, since $p_{j}$ has $b_{j}$ preimages under $f$ (the $p_{j}$ 's are chosen generically), there are $b_{j}$ choices for the location of $x_{j}$, the marked point on $G_{j}$, for each homomorphism $f: G_{j} \rightarrow F_{j}$. Thus the data $\Lambda(\mathbf{b})$ completely determines $f$ restricted to $G_{1}, \ldots, G_{g}$. Now $\left.f\right|_{S}$ is determined and so we can reconstruct $f$ completely from $\left.f\right|_{N_{1}}, \ldots,\left.f\right|_{N_{24}}$ and $\Lambda(\mathbf{b})$. It follows that the subset of $\overline{\mathcal{M}}_{\mathbf{a}, \mathbf{b}}$ with fixed $\Lambda(\mathbf{b})$ is isomorphif 5 to the product of the moduli spaces $\prod_{i=1}^{24} \overline{\mathcal{M}}_{\left[a_{i}\right]_{i}, 0}$, where $[c]_{i}$ denotes the 24 -tuple $(0, \ldots, c, \ldots, 0)$ with $c$ in the $i$ th slot and zeros elsewhere. The connected components of $\overline{\mathcal{M}}_{\mathbf{a}, \mathbf{b}}$ are in one-to-one correspondence with the data $(\Lambda(\mathbf{b}), s(\mathbf{a}))$ and Theorem 5.1 is proved provided we can show that the connected components of $\overline{\mathcal{M}}_{[a], 0}$ are in one-to-one correspondence with admissible sequences $s$ of magnitude $|s|=a$. We state this as

Lemma 5.3. Let $\overline{\mathcal{M}}_{a}$ be the moduli space of stable, genus 0 maps to $X$ with image $S+a N$ for any fixed nodal fiber $N$. Then $\overline{\mathcal{M}}_{a}$ is a disjoint union $\coprod_{s(a)} \overline{\mathcal{M}}_{s(a)}$ of spaces $\overline{\mathcal{M}}_{s(a)}$ labeled by admissible sequences $s(a)=\left\{s_{n}(a)\right\}$ with $|s(a)|=$ $\sum_{n} s_{n}(a)=a$.

Proof. Let $\Sigma(a)$ be a genus 0 nodal curve consisting of a linear chain of $2 a+1$ smooth components $\Sigma_{-a}, \ldots, \Sigma_{a}$ with an additional smooth component $\Sigma_{*}$ meeting $\Sigma_{0}$ (so $\Sigma_{n} \cap \Sigma_{m}=\emptyset$ unless $|n-m|=1$ and $\Sigma_{*} \cap \Sigma_{n}=\emptyset$ unless $n=0$ ). Fix a map of

$$
h: \Sigma(a) \rightarrow X
$$

with image $S \cup N$ in the following way. Map $\Sigma_{*}$ to $S$ with degree 1 and map each $\Sigma_{n}$ to $N$ with degree one. Require that a neighborhood of each singular point $\Sigma_{n} \cap \Sigma_{n+1}$ is mapped biholomorphically onto its image with $\Sigma_{n} \cap \Sigma_{n+1}$ mapping to the nodal point of $N$ so that $h$ is a local embedding.

Let $\left\{s_{n}(a)\right\}$ be an admissible sequence with $|s(a)|=a$. Since the index $n$ of the sequence cannot be smaller than $-a$ or larger than $a$, we can extend $\left\{s_{n}(a)\right\}$ to a sequence $s_{-a}, \ldots, s_{a}$ by setting $s_{n}=0$ for those not previously defined. Define $\overline{\mathcal{M}}_{s(a)}$ to be the moduli space of genus 0 stable maps with $\Sigma(a)$ as the target in the class

$$
\Sigma_{*}+\sum_{n=-a}^{a} s_{n}(a) \Sigma_{n}
$$

\footnotetext{
${ }^{5}$ Strictly speaking, we have only shown that the isomorphism is an isomorphism as coarse moduli spaces. However, it follows from Theorem B.1 of Appendix B that we get an isomorphism of schemes/stacks/moduli functors. This is discussed further in the next subsection.
} 


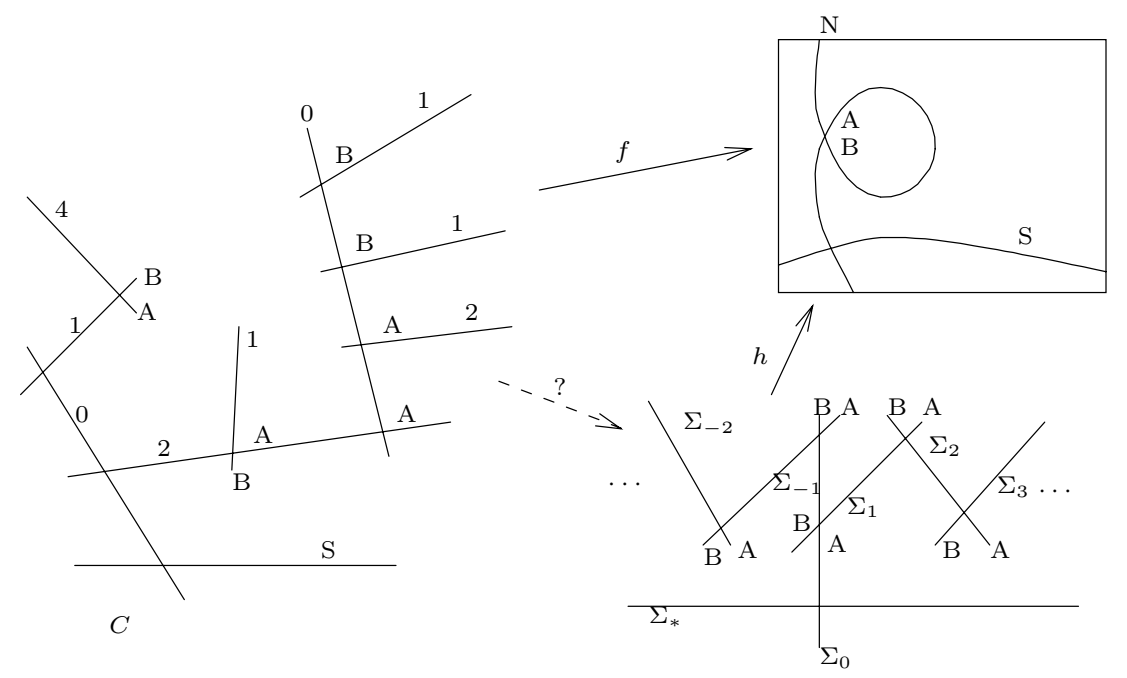

FIGURE 2.

By composition with the fixed map $h$ from $\Sigma(a)$ to $X$, we get a stable map in $\overline{\mathcal{M}}_{a}$ from each map in $\overline{\mathcal{M}}_{s(a)}$. To prove the lemma we need to show 6 that every map in $\overline{\mathcal{M}}_{a}$ factors uniquely in this way through a map in $\overline{\mathcal{M}}_{s(a)}$ for some admissible $s(a)=\left\{s_{n}(a)\right\}$. Figure 2 illustrates some of the phenomena that can occur. The numbers on components of $C$ indicate the degree of $f$ on that component. The $A$ 's and $B$ 's indicate the local behavior of the map when a nodal point of $C$ is mapped to the nodal point in $N$.

Consider the dual graph of the domain $C$ of a map $f: C \rightarrow X$ in $\overline{\mathcal{M}}_{a}$. The graph is a tree with one special vertex $v_{*}$ (the component mapping to $S$ ) whose valence is 1 . Every other vertex $v$ is marked with a non-negative integer $l_{v}$ (the degree of the component associated to $v$ ) such that the sum of the $l_{v}$ 's is $a$. Vertices with a marking of 0 (collapsed components) must have valence at least three (stability). We mark the edges in the following way. Each edge corresponds to a nodal singularity in $C$ and if the node is not mapped to the nodal point in $N$, we do not mark the edge. The remaining edges are marked with either a pair of the letters $A$ or $B$, a single letter of $A$ or $B$, or nothing as follows. Label the two branches near the node in $N$ by $A$ and $B$. Three things can then happen for an edge corresponding to a node in $C$ that gets mapped to the node in $N$.

1. If the edge connects two collapsed components, do not mark the edge.

2 . If the edge connects one collapsed component with one non-collapsed component, mark the edge with an $A$ or $B$ depending on whether the non-collapsed component is mapped (locally) to the $A$ branch or the $B$ branch.

3. Finally, if the edge connects two non-collapsed components, then mark the edge with two of the letters $A$ or $B$, one near each of the vertices, according to which branch that corresponding component maps to (locally). Note that all the combinations $A B, B A, A A$, and $B B$ can occur.

\footnotetext{
${ }^{6}$ To prove the lemma as stated we also need to show that $\overline{\mathcal{M}}_{s(a)}$ is connected. This is not hard, but since we never actually use this part of the result, we will leave its proof to the reader.
} 


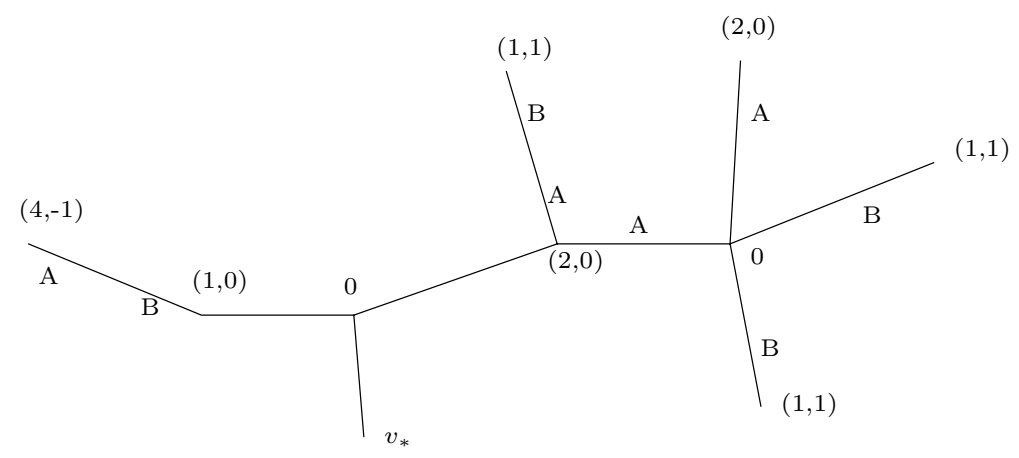

Figure 3.

The markings on our graph now tell us how and when the map "jumps" branches. Jumping from $A$ to $B$ will correspond to moving from $\Sigma_{n}$ to $\Sigma_{n+1}$ in the factored map. To determine which component $\Sigma_{n}$ a component of $C$ gets mapped to, we count how many "jumps" occur between the corresponding vertex $v$ and the central vertex $v_{*}$. For every non-collapsed component vertex $v$ assign its index $n_{v}$ by traveling from $v_{*}$ to $v$ in the graph and counting +1 for each $A B$ pair passed through, -1 for each $B A$ pair, and 0 for each $A A$ or $B B$ pair. We can now uniquely factor $f: C \rightarrow X$ through the fixed map $\Sigma(a) \rightarrow X$. The component of $C$ corresponding to a vertex $v$ gets mapped to $\Sigma_{n_{v}}$. The factorization is unique since away from the $A B$ or $B A$ jumps, $f$ factors uniquely through the normalization of $N$.

The marked dual graph for the previously illustrated example is given in Figure 3 . Here we've marked the vertices with $\left(l_{v}, n_{v}\right)$ so in this example $s_{-1}=4, s_{0}=5$, and $s_{1}=3$.

5.2. Obstruction theory and virtual classes. In this subsection we show that the virtual class defining the contribution of $\overline{\mathcal{M}}_{s, \Lambda}$ to $N_{g}(n)$ is the product of virtual classes defined on $\overline{\mathcal{M}}_{s\left(a_{i}\right)}$. This is the most technical subsection of the paper, but it is essentially self-contained, and can be skipped by casual readers.

To compute the contribution of $\overline{\mathcal{M}}_{s, \Lambda}$ to $N_{g}(n)$, we recall the definition of the invariant. Let $\overline{\mathcal{M}}_{g, g}(X, T ; C)$ be the moduli space of $g$-marked, genus $g$ stable maps to $X$ (with its twistor family) in the class of $C$. Let $\overline{\mathcal{M}}(X, \mathbf{p}) \subset \overline{\mathcal{M}}_{g, g}(X, T ; C)$ denote the restriction to those maps that send the $j$ th marked point to the point $p_{j} \in X$; the virtual dimension of $\overline{\mathcal{M}}(X, \mathbf{p})$ is 0 . By definition, $N_{g}(n)$ is the evaluation of $e^{*}\left(\left[p_{1}\right]^{\vee} \otimes \cdots \otimes\left[p_{g}\right]^{\vee}\right)$ on $\left[\overline{\mathcal{M}}_{g, g}(X, T ; C)\right]^{\text {vir }}$. Alternatively, one can construct a 0 -dimensional virtual class directly on the cut-down moduli space $[\overline{\mathcal{M}}(X, \mathbf{p})]^{\text {vir }}$, which gives an invariant coinciding with the above definition (see Proposition A.4 in Appendix $(\mathrm{A})$; this is the tack we take.

Virtual classes in Gromov-Witten theory were constructed in the symplectic category by Li-Tian [28] and Siebert [33] and in the algebraic category by Li-Tian [27] and Behrend-Fantechi [5], [6]. The algebraic and symplectic versions were shown to coincide by Li-Tian [26] and Siebert [34]. This enables us to compute the invariant purely algebro-geometrically, although we need the machinery of the symplectic category to define it. 
To understand the class $[\overline{\mathcal{M}}(X, \mathbf{p})]^{v i r}$ in a purely algebraic way, we use the fact that, although the twistor family is not algebraic, it is an analytic family. In his proof that the algebraic and symplectic versions of Gromov-Witten invariants are the same, Siebert constructs an analytic version of Behrend and Fantechi's virtual class. We can apply this construction directly to the twistor family. Let

$$
\pi: Z \rightarrow T
$$

denote the twistor family. Topologically $Z=X \times T$ but we give the fibers $Z_{t}=$ $\pi^{-1}(t)$ the Kähler structure $\left(X, J_{t}, \omega_{t}\right)$. Let $t_{0}$ be the unique member of $T$ for which $\left(X, J_{t_{0}}, \omega_{t_{0}}\right)$ admits a holomorphic curve in the class $[C]$ and let $X$ denote the (algebraic) space $Z_{t_{0}}$.

$\overline{\mathcal{M}}(X, \mathbf{p})$ is a moduli space over $\mathfrak{M}_{g, g} \times T$ where $\mathfrak{M}_{g, g}$ is the moduli stack of $g$-marked genus $g$ pre-stable curves. The virtual class $[\mathcal{M}(X, \mathbf{p})]^{\text {vir }}$ is determined as in Proposition A.4 by the perfect relative obstruction theory

$$
\left[R^{\bullet} \pi_{*} f^{*}\left(T_{Z / T}(-\mathbf{x})\right)\right]^{\vee} \rightarrow L_{\overline{\mathcal{M}}(X, \mathbf{p}) / \mathfrak{M}_{g, g} \times T} .
$$

This requires some explanation. If $Z / T$ were a family in the algebraic category we would say the following: The above map is a morphism in the derived category of coherent sheaves on $\overline{\mathcal{M}}(X, \mathbf{p}) / \mathfrak{M}_{g, g} \times T$ and $L_{\overline{\mathcal{M}}(X, \mathbf{p}) / \mathfrak{M}_{g, g} \times T}$ is the cotangent complex of $\overline{\mathcal{M}}(X, \mathbf{p})$ relative to $\mathfrak{M}_{g, g} \times T$. $T_{Z / T}$ is the relative tangent bundle of $Z / T$ and $-\mathbf{x}=-x_{1}-\cdots-x_{g}$ where $x_{j}$ is the divisor on the universal family over $\overline{\mathcal{M}}_{g, g}(X, T ; C)$ corresponding to the $j$ th marked point. The term perfect means that the induced map in cohomology is an isomorphism on $h^{0}$ and surjective on $h^{-1}$. Furthermore, the left hand side of (2) should be equivalent in the derived category to a two-term complex of bundles. Note that the moduli stack $\mathfrak{M}_{g, g}$ of pre-stable curves is an Artin stack (not of Deligne-Mumford or even finite type); the Deligne-Mumford stack of stable curves $\overline{\mathcal{M}}_{g, g}$ is an open substack. The map from $\overline{\mathcal{M}}(X, \mathbf{p})$ to $\mathfrak{M}_{g, g}$ is given by $(f: C \rightarrow X) \mapsto C$ (and not stabilizing $C$ ).

Since $Z / T$ is not algebraic but analytic, we wish to use Siebert's analytic reformulation of the virtual class. Since the analogues of Artin stacks in the analytic category are not well developed in the literature, Siebert avoids them by working locally and then globalizing. Locally, there is a rigidification trick so that the map (2) and resulting virtual class can be constructed using the derived category of coherent analytic orbi-sheaves on analytic orbi-spaces (see section 1.2 of [34]). Rather than introduce excessive notation, we will write as if the constructions were already global and $T$ were algebraic as we have in Equation (2).

The object $E^{\bullet}=\left[R^{\bullet} \pi_{*} f^{*}\left(T_{Z / T}(-\mathbf{x})\right)\right]^{\vee}$ is the obstruction theory. It is represented by a two-term complex in degrees -1 and 0 whose cohomology gives sheaves whose stalks over a map $\{f:(C, \mathbf{x}) \rightarrow X\}$ are the duals to the deformation and obstruction spaces:

$$
\begin{aligned}
& H^{0}\left(C, f^{*}(T X(-\mathbf{x}))\right)^{\vee}, \\
& H^{1}\left(C, f^{*}(T X(-\mathbf{x}))\right)^{\vee} .
\end{aligned}
$$

The $H^{0}$ space is the space of infinitesimal deformations of the map preserving the condition that $f\left(x_{j}\right)=p_{j}$ and the $H^{1}$ space is the space of obstructions to those deformations.

In the previous subsection we showed that the connected components of the moduli space $\overline{\mathcal{M}}(X, \mathbf{p})$ are indexed by the tuples $(\mathbf{a}, \mathbf{b})$ and the data $(s(\mathbf{a}), \Lambda(\mathbf{b}))$. 
Furthermore, we identified each component with a product:

$$
\begin{aligned}
\overline{\mathcal{M}}(X, \mathbf{p}) & \cong \coprod_{\mathbf{a}, \mathbf{b}} \coprod_{s, \Lambda} \overline{\mathcal{M}}_{s(\mathbf{a}), \Lambda(\mathbf{b})} \\
& \cong \coprod_{\mathbf{a}, \mathbf{b}} \coprod_{s, \Lambda}\left(\prod_{i=1}^{24} \overline{\mathcal{M}}_{s\left(a_{i}\right)}\right)\left(\prod_{j=1}^{g} \overline{\mathcal{M}}_{\Lambda\left(b_{j}\right)}\right) .
\end{aligned}
$$

Here $\overline{\mathcal{M}}_{\Lambda\left(b_{j}\right)}$ is just a point corresponding to the unique map $f: S \cup G_{j} \rightarrow X$ that is the identity on $S$ and the unique $b_{j}$-fold cover of $F_{j}$ corresponding to the data $\Lambda\left(b_{j}\right)$.

Strictly speaking, we have only proven that the second isomorphism above is an isomorphism of coarse moduli spaces. However, it follows from Theorem B.1 of Appendix $\mathrm{B}$ that the isomorphism is an isomorphism of stacks.

We wish to show that the virtual class $\left[\overline{\mathcal{M}}_{s, \Lambda}\right]^{\text {vir }}$ is a product of virtual classes coming from the factors $\overline{\mathcal{M}}_{s\left(a_{i}\right)}$ and $\overline{\mathcal{M}}_{\Lambda\left(b_{j}\right)}$. To do this, we need to convert the relative obstruction theory defining $\left[\overline{\mathcal{M}}_{s, \Lambda}\right]^{\text {vir }}$ into a relative theory that is compatible with the product structure. Since we know that all the domains of the maps in $\overline{\mathcal{M}}_{s, \Lambda}$ have a particular degeneration type and we know that there is only one member $t_{0} \in T$ for which the corresponding moduli space is non-empty, we can factor the map

$$
\tau: \overline{\mathcal{M}}_{s, \Lambda} \rightarrow \mathfrak{M}_{g, g} \times T
$$

through a smooth local embedding $i_{\rho^{\sharp}}$ :

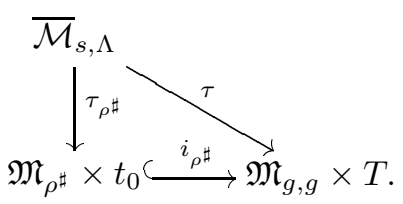

We define $\mathfrak{M}_{\rho^{\sharp}}$ as follows. First, there is a smooth stack $\mathfrak{M}_{\rho}$ and a local embedding $\mathfrak{M}_{\rho} \hookrightarrow \mathfrak{M}_{g, g}$ for every modular graph of genus $g$ with $g$ tails (see BehrendManin [7] for the terminology). Let $\rho$ be the graph with one central vertex $S$ of genus 0,24 genus 0 vertices $D_{1}, \ldots, D_{24}$ connected to $S$, and $g$ genus 1 vertices $G_{1}, \ldots, G_{g}$ connected to $S$, each with one tail. $7 \mathfrak{M}_{\rho} \subset \mathfrak{M}_{g, g}$ is the local substack of genus $g, g$-marked curves with degeneration type determined by $\rho$. Let $\sigma$ be the modular graph obtained by cutting all the edges of $\rho$. $\sigma$ is a disconnected graph with 24 components $\alpha_{1}, \ldots, \alpha_{24}$ consisting of a genus 0 vertex with a tail, $g$ components $\beta_{1}, \ldots, \beta_{g}$ consisting of a genus 1 vertex with 2 tails, and one component $\sigma_{S}$ consisting of a genus zero vertex with $24+g$ tails as in Figure 4.

It follows from the properties of modular graphs and their associated moduli stacks (see [7]) that there are isomorphisms

$$
\mathfrak{M}_{\rho} \cong \mathfrak{M}_{\sigma} \cong \mathfrak{M}_{\sigma_{S}} \times \prod_{i=1}^{24} \mathfrak{M}_{\alpha_{i}} \prod_{j=1}^{g} \mathfrak{M}_{\beta_{j}}
$$

Note that $\mathfrak{M}_{\sigma_{S}} \cong \mathfrak{M}_{0,24+g}$ and let $p_{S} \in \mathfrak{M}_{0,24+g}$ be the point corresponding to the fixed (marked) curve $(S, \mathbf{y}, \mathbf{z})=\left(S, y_{1}, \ldots, y_{24}, z_{1}, \ldots, z_{g}\right) \subset X$. Let $\mathfrak{M}_{\rho^{\sharp}} \subset \mathfrak{M}_{\rho}$

\footnotetext{
${ }^{7}$ If some of the $a_{i}$ are 0 , then $\rho$ should not have the corresponding vertices; those cases work exactly the same in every other respect, so we will assume for notational convenience that all the $a_{i}$ 's are non-zero.
} 


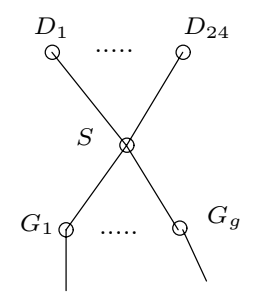

$\rho$

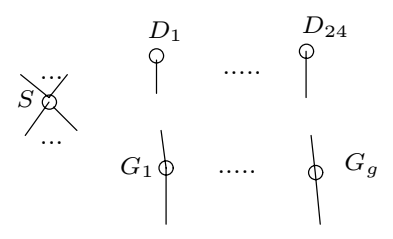

$\sigma$

Figure 4.

parameterize curves with the degeneration type $\rho$ and with the curve corresponding to the vertex $S$ fixed to be the marked curve $(S, \mathbf{y}, \mathbf{z})$, i.e.

$$
\mathfrak{M}_{\rho^{\sharp}} \cong p_{S} \times \prod_{i=1}^{24} \mathfrak{M}_{\alpha_{i}} \prod_{j=1}^{g} \mathfrak{M}_{\beta_{j}} .
$$

Similarly, by gluing a rational curve with a fixed parameterization $\mathbf{P}^{1} \rightarrow(S, \mathbf{y}, \mathbf{z})$ to the marked point of a curve in $\mathfrak{M}_{\alpha_{i}}$ at the point $y_{i}$ we obtain isomorphisms

$$
\mathfrak{M}_{\alpha_{i}^{\sharp}} \cong \mathfrak{M}_{\alpha_{i}}
$$

so that, by definition, $\mathfrak{M}_{\alpha_{i}^{\sharp}}$ parameterizes genus 0 curves with a component canonically isomorphic to $(S, \mathbf{y}, \mathbf{z})$ and all other components attached to $S$ at the fixed point $y_{i} \in S$. In a similar manner we get the stack $\mathfrak{M}_{\beta_{j}^{\sharp}} \cong \mathfrak{M}_{\beta_{j}}$.

Since the domain $C$ of every map $f: C \rightarrow X$ in $\overline{\mathcal{M}}_{s, \Lambda}$ lies in $\mathfrak{M}_{\rho^{\sharp}}$ and, by Theorem B.1 of Appendix B, there are no infinitesimal deformations of $f: C \rightarrow X$ that deform $C$ out of $\mathfrak{M}_{\rho^{\sharp}}$, the factorization given by diagram (3) exists.

We obtain similar factorizations for the moduli stacks $\overline{\mathcal{M}}_{s\left(a_{i}\right)}$ and $\overline{\mathcal{M}}_{\Lambda\left(b_{j}\right)}$ :

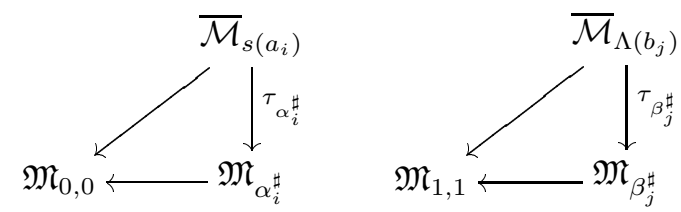

Let $\pi: \mathcal{C}_{s, \Lambda} \rightarrow \overline{\mathcal{M}}_{s, \Lambda}$ be the universal curve and let $f: \mathcal{C}_{s, \Lambda} \rightarrow X$ be the universal map. Let $\mathcal{S}, \mathcal{D}_{i}$, and $\mathcal{G}_{j}$ be the components of the universal curve corresponding to the components $S, D_{i}$, and $G_{j}$ discussed in the previous subsection. Note that $\mathcal{S} \cong S \times \mathfrak{M}_{s, \Lambda}$ with $\left.\pi\right|_{\mathcal{S}}$ projection on the second factor and $\left.f\right|_{\mathcal{S}}$ projection on the first factor followed by the inclusion $S \subset X$. The universal curve $\mathcal{C}_{s, \Lambda}$ has sections $y_{i}$ and $z_{j}$ corresponding to the intersections $\mathcal{S} \cap \mathcal{D}_{i}$ and $\mathcal{S} \cap \mathcal{G}_{j}$ respectively. Let $p$ denote the map from the disjoint union of $\mathcal{S}, \mathcal{D}_{1}, \ldots, \mathcal{D}_{24}, \mathcal{G}_{1}, \ldots, \mathcal{G}_{g}$ to $\mathcal{C}_{s, \Lambda}$ obtained by gluing along $y_{i}$ and $z_{j}$. The inverse image of $y_{i}$ and $z_{j}$ under the gluing map $p$ are denoted $y_{i}^{\prime}, y_{i}^{\prime \prime}$ and $z_{j}^{\prime}, z_{j}^{\prime \prime}$ respectively where the double primed sections lie in $\mathcal{S}$ and the single primed sections lie in $\mathcal{D}_{i}$ and $\mathcal{G}_{j}$. Let $\pi^{\prime}$ denote the composition $\pi \circ p$. 
In summary, we get the following diagram:

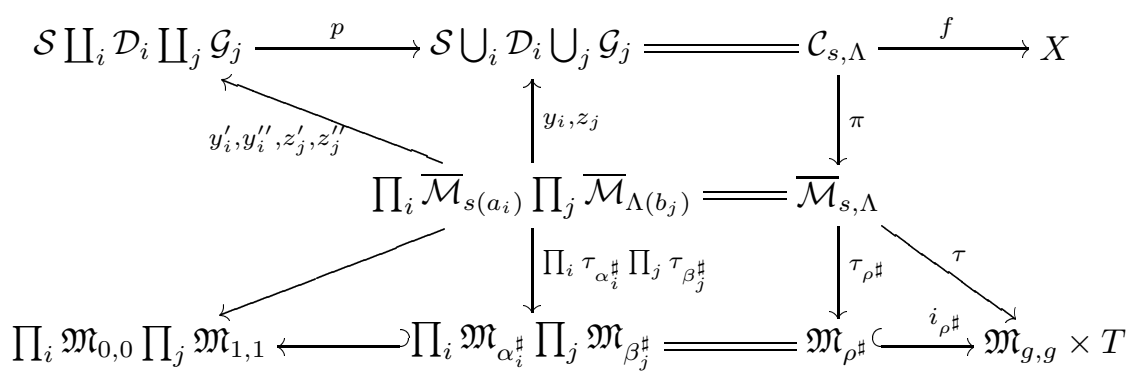

Recall that the virtual class $\left[\overline{\mathcal{M}}_{s, \Lambda}\right]^{v i r}$ is determined by the perfect relative obstruction theory $\left[R^{\bullet} \pi_{*}\left(f^{*} T_{X} \otimes \mathcal{O}(-\mathbf{x})\right)\right]^{\vee}$ which we denote by $E^{\bullet}$. From Proposition A.1 of Appendix $\mathrm{A}$, we get an object $F^{\bullet}$ fitting into a morphism of triangles

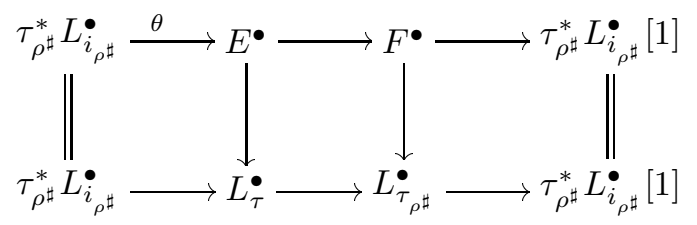

defining a perfect relative obstruction theory $F^{\bullet} \rightarrow L_{\tau_{\rho^{\sharp}}}^{\bullet}$ determining the same virtual class. We use the obstruction theory $F^{\bullet}$ because it is more compatible with the product structure of $\overline{\mathcal{M}}_{s, \Lambda}$.

Before we can make this more explicit, we need another exact triangle for $E^{\bullet}$ that arises from the component structure of $\mathcal{C}_{s, \Lambda}$.

Let $W$ be the bundle $f^{*}\left(T_{X}\right) \otimes \mathcal{O}(-\mathbf{x})$ on $\mathcal{C}_{s, \Lambda}$. We apply $p_{*}$ to the morphisms

$$
p^{*} W \rightarrow y_{i *}^{\prime} y_{i}^{\prime *} p^{*} W
$$

and, noting that $p \circ y_{i}^{\prime}=y_{i}$, we obtain morphisms

$$
\varphi_{y_{i}^{\prime}}: p_{*} p^{*} W \rightarrow y_{i *} y_{i}^{*} W
$$

Similarly, there are morphisms

$$
\begin{aligned}
& \varphi_{y_{i}^{\prime \prime}}: p_{*} p^{*} W \rightarrow y_{i *} y_{i}^{*} W \\
& \varphi_{z_{j}^{\prime}}: p_{*} p^{*} W \rightarrow z_{j *} z_{j}^{*} W \\
& \varphi_{z_{j}^{\prime \prime}}: p_{*} p^{*} W \rightarrow z_{j *} z_{j}^{*} W
\end{aligned}
$$

Let $\varphi_{y_{i}}=\varphi_{y_{i}^{\prime \prime}}-\varphi_{y_{i}^{\prime}}$ and $\varphi_{z_{j}}=\varphi_{z_{j}^{\prime \prime}}-\varphi_{z_{j}^{\prime}}$ and then let

$$
\varphi=\oplus_{i} \varphi_{y_{i}} \oplus_{j} \varphi_{z_{j}}
$$

We then have an exact sequence of sheaves on $\mathcal{C}_{s, \Lambda}$ (cf. Behrend [5], pg. 608)

$$
0 \longrightarrow W \longrightarrow p_{*} p^{*} W \stackrel{\varphi}{\longrightarrow} \oplus_{i} y_{i *} y_{i}^{*} W \oplus_{j} z_{j *} z_{j}^{*} W \longrightarrow 0 .
$$

Apply $R^{\bullet} \pi_{*}$ to this sequence and recall that $\pi^{\prime}=\pi \circ p$ and that $y_{i}$ and $z_{j}$ are sections. We obtain a triangle

$$
R^{\bullet} \pi_{*}\left(f^{*} T_{X}(-\mathbf{x})\right) \longrightarrow R^{\bullet} \pi_{*}^{\prime} p^{*}\left(f^{*} T_{X}(-\mathbf{x})\right) \stackrel{R \pi_{*}(\varphi)}{\longrightarrow} \oplus_{i} y_{i}^{*} W \oplus_{j} z_{j}^{*} W .
$$


We denote the restriction of $f$ to the components of $\mathcal{C}_{s, \Lambda}$ by $f_{S}, f_{D_{i}}$, and $f_{G_{j}}$ and we let $E_{S}^{\bullet}, E_{D_{i}}^{\bullet}$, and $E_{G_{j}}^{\bullet}$ denote the objects $\left[R^{\bullet} \pi_{*} f_{S}^{*} T_{X}\right]^{\vee},\left[R^{\bullet} \pi_{*} f_{D_{i}}^{*} T_{X}\right]^{\vee}$, and $\left[R^{\bullet} \pi_{*}\left(f_{G_{j}}^{*} T_{X}\left(-x_{j}\right)\right)\right]^{\vee}$ respectively. Dualizing the above triangle, we get

$$
\oplus_{i} y_{i}^{*} W^{\vee} \oplus_{j} z_{j}^{*} W^{\vee} \longrightarrow E_{S}^{\bullet} \oplus_{i} E_{D_{i}}^{\bullet} \oplus_{j} E_{G_{j}}^{\bullet} \longrightarrow E^{\bullet} .
$$

Note that the induced long exact sequence in cohomology is an exact sequence of sheaves on $\overline{\mathcal{M}}_{s, \Lambda}$ which on the stalks over $(f: C \rightarrow X)$ is the long exact sequence associated to the normalization of $C$ at the points $y_{i}$ and $z_{j}$.

Lemma 5.4. The exact triangles (5) and (6) fit into the following commuting diagram where the rows and columns are exact triangles and the objects $F_{D_{i}}^{\bullet}$ and $F_{G_{j}}^{\bullet}$ are pulled back from $\overline{\mathcal{M}}_{s\left(a_{i}\right)}$ and $\overline{\mathcal{M}}_{\Lambda\left(b_{j}\right)}$ (the direct sums are over the indices $i$ and j).

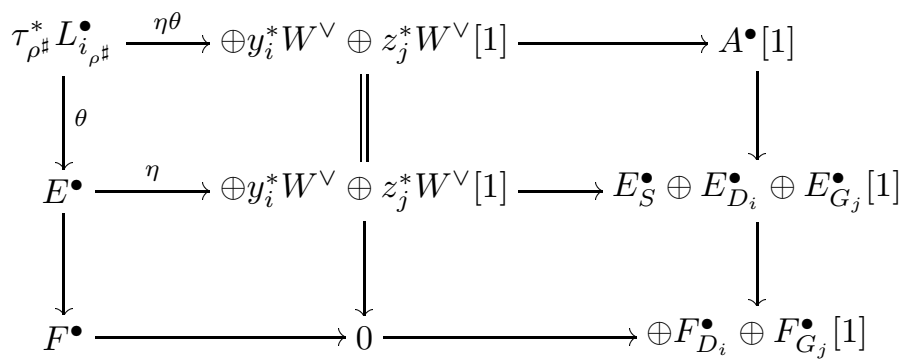

In particular, the bottom row implies $F^{\bullet}=\oplus F_{D_{i}}^{\bullet} \oplus F_{G_{j}}^{\bullet}$.

Proof. The morphisms $\theta$ and $\eta$ come from the triangles (50) and (6) respectively and they uniquely determine the triangles. The upper left square can then be uniquely completely to the above diagram (Weibel [35], page 378).

To see that the object in the lower right corner of the diagram that we obtain is a direct sum of the form $\oplus F_{D_{i}}^{\bullet} \oplus F_{G_{j}}^{\bullet}$ we need to understand a little about the morphisms involved, in particular $\eta \theta$.

Roughly speaking, the main point is that everything in the above diagram splits over $i$ and $j$ except $E_{S}^{\bullet}$ and corresponding pieces of $\tau_{\rho^{\sharp}}^{*} L_{i_{\rho^{\sharp}}}$ which then appear in $A^{\bullet}$ to cancel the $E_{S}^{\bullet}$ term.

In general, a morphism in the derived category is not determined by the induced morphisms in cohomology. However, the objects $\tau_{\rho^{\sharp}}^{*} L_{i_{\rho^{\sharp}}}^{\bullet}$ and $\oplus y_{i}^{*} W^{\vee} \oplus z_{j}^{*} W^{\vee}[1]$ have non-zero cohomology in only degree -1 , and so they are determined by their cohomology and the morphism $\eta \theta$ is determined by the map in cohomology (Proposition 4.3 of [22]). Thus, abbreviating $y_{i}^{*} W$ and $z_{j}^{*} W$ by $W_{i}$ and $W_{j}$, we have

$$
A^{\bullet}=\left[\oplus W_{i} \oplus W_{j} \rightarrow\left(\tau_{\rho^{\sharp}}^{*} L_{i_{\rho^{\sharp}}^{-1}}^{-1}\right]^{\vee}\right.
$$

where the map is $\left(h^{-1}(\eta \theta)\right)^{\vee}$, the dual of the induced map in cohomology. Here $W_{i}, W_{j}$, and $\tau_{\rho^{\sharp}}^{*} L_{\rho_{\rho^{\sharp}}}^{-1}$ are sheaves on $\overline{\mathcal{M}}_{s, \Lambda}$ which are readily identified.

$W_{i}$ is the trivial rank 2 bundle on $\overline{\mathcal{M}}_{s, \Lambda}$ whose fibers are canonically $T_{y_{i}} X$, and $W_{j}$ is the trivial rank 2 bundle whose fibers are canonically identified with $T_{z_{j}} X$. In general, we denote trivial bundles on $\overline{\mathcal{M}}_{s, \Lambda}$ by their fibers so that, for example, we just write $T_{y_{i}} X$ for $W_{i}=T_{y_{i}} X \otimes \mathcal{O}_{\overline{\mathcal{M}}_{s, \Lambda}}$. The bundle $\left(\tau_{\rho^{\sharp}}^{*} L_{i_{\rho^{\sharp}}}^{-1}\right)^{\vee}$ is the pullback 
of $N_{\rho^{\sharp}}$, the normal bundle of $\mathfrak{M}_{\rho^{\sharp}} \times t_{0} \hookrightarrow \mathfrak{M}_{g, g} \times T$. It fits into an exact sequence coming from $\mathfrak{M}_{\rho^{\sharp}} \times t_{0} \hookrightarrow \mathfrak{M}_{\rho} \times T \hookrightarrow \mathfrak{M}_{g, g} \times T$, namely

$$
0 \rightarrow \operatorname{Def}(S, \mathbf{y}, \mathbf{z}) \oplus T^{0} \rightarrow N_{\rho^{\sharp}} \rightarrow N_{\rho} \rightarrow 0
$$

where $\operatorname{Def}(S, \mathbf{y}, \mathbf{z})=T_{p_{S}} \mathfrak{M}_{0,24+g}$ is the tangent space to $\mathfrak{M}_{0,24+g}$ at the point $p_{S}$ corresponding to $(S, \mathbf{y}, \mathbf{z})$ and $T^{0}$ denotes $T_{t_{0}} T$, the tangent space to the twistor family at $t_{0}$. The bundle $N_{\rho}$ is the normal bundle of the local embedding $\mathfrak{M}_{\rho} \hookrightarrow$ $\mathfrak{M}_{g, g}$. The fibers of the bundle $N_{\rho}$ classify infinitesimal smoothings of the nodes $y_{i}$ and $z_{j}$, i.e.

$$
\oplus_{i}\left(T_{y_{i}} S \otimes T_{y_{i}} D_{i}\right) \oplus_{j}\left(T_{z_{j}} S \otimes T_{z_{j}} G_{j}\right) .
$$

The vector space $\operatorname{Def}(S, \mathbf{y}, \mathbf{z})$ is naturally given as a quotient:

$$
0 \rightarrow \text { Aut } S \rightarrow \oplus T_{y_{i}} S \oplus T_{z_{j}} S \rightarrow \operatorname{Def}(S, \mathbf{y}, \mathbf{z}) \rightarrow 0 .
$$

It can be seen that this, together with the previous exact sequence, combine to express $\tau_{\rho^{\sharp}}^{*} N_{\rho^{\sharp}}$ as a quotient of the form

$$
0 \rightarrow \text { Aut } S \rightarrow T^{0} \oplus M_{i} \oplus M_{j} \rightarrow \tau_{\rho^{\sharp}}^{*} N_{\rho^{\sharp}} \rightarrow 0
$$

where $M_{i}$ and $M_{j}$ are bundles pulled back from $\overline{\mathcal{M}}_{s\left(a_{i}\right)}$ and $\overline{\mathcal{M}}_{\Lambda\left(b_{j}\right)}$ respectively.

The map

$$
h^{-1}(\eta \theta)^{\vee}: \oplus W_{i} \oplus W_{j} \rightarrow \tau_{\rho^{\sharp}}^{*} N_{\rho^{\sharp}}
$$

can be explicitly identified via geometry and obstruction theory (see Appendix B). We only need to understand this map to the extent to which it is compatible with the product structure. At the stalks over $(f: C \rightarrow X)$, the map $h^{-1}(\eta \theta)^{\vee}$ is the composition

$$
\left.\oplus H^{0}\left(y_{i}, f^{*} T_{X}\right) \oplus H^{0}\left(z_{j}, f^{*} T_{X}\right) \rightarrow H^{1}\left(C, f^{*} T_{X} \otimes \mathcal{O}(-\mathbf{x})\right) \rightarrow N_{\rho^{\sharp}}\right|_{C} .
$$

Here the first map is the connecting homomorphism in the long exact sequence coming from normalizing the double points $y_{i}$ and $z_{j}$ (i.e. it is the dual of the induced map $\left.h^{-1}(\eta)\right)$. The second map is the splitting of Theorem B.1 from Appendix B (see Corollary B.2). From the proof of that theorem we see that the map $h^{-1}(\eta \theta)^{\vee}$ lifts to a map $\oplus W_{i} \oplus W_{j} \rightarrow M_{i} \oplus M_{j}$ which is a product of maps $W_{i} \rightarrow M_{i}$ and $W_{j} \rightarrow M_{j}$. Thus we have a quasi-isomorphism:

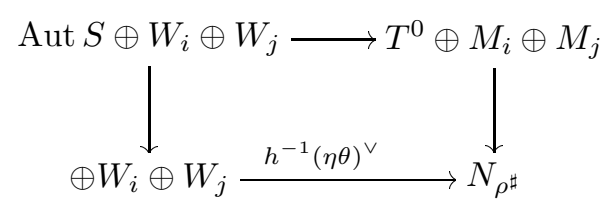

and so we see that

$$
A^{\bullet}=\left[\text { Aut } S \oplus W_{i} \oplus W_{j} \rightarrow T^{0} \oplus M_{i} \oplus M_{j}\right]^{\vee} .
$$

Now consider the object $E_{S}^{\bullet}$. Since $\mathcal{S} \cong \overline{\mathcal{M}}_{s, \Lambda} \times S$ and $\pi$ is just projection onto the first factor and $f$ is just projection onto the second factor followed by the inclusion $S \hookrightarrow X$, the object $E_{S}^{\bullet}$ is just $\left[H^{0}(S, T X) \stackrel{0}{\rightarrow} H^{1}(S, T X)\right]^{\vee}$ where $H^{i}(S, T X)$ denotes the trivial bundle with the corresponding fiber. Note that since 
$\left.T X\right|_{S}=T S \oplus N S \cong \mathcal{O}_{S}(2) \oplus \mathcal{O}_{S}(-2)$, we see that $H^{0}(S, T X)=H^{0}(S, T S)=$ Aut $S$ and $H^{1}(S, T X)=H^{1}(S, N S)$. Thus we can express $E_{S}^{\bullet}$ as

$$
E_{S}^{\bullet} \cong\left[\text { Aut } S \stackrel{0}{\longrightarrow} H^{1}(S, N S)\right]^{\vee}
$$

Finally, to finish the proof of Lemma 5.4 we show that the mapping cone of the morphism $A^{\bullet} \rightarrow E_{S}^{\bullet} \oplus E_{D_{i}}^{\bullet} \oplus E_{G_{j}}^{\bullet}$, i.e.

[Aut $\left.S \oplus W_{i} \oplus W_{j} \rightarrow T^{0} \oplus M_{i} \oplus M_{j}\right]^{\vee} \rightarrow\left[\text { Aut } S \rightarrow H^{1}(S, N S)\right]^{\vee} \oplus E_{D_{i}}^{\bullet} \oplus E_{G_{j}}^{\bullet}$,

is quasi-isomorphic to the direct sum of the mapping cones of morphisms $\left[W_{i} \rightarrow\right.$ $\left.M_{i}\right]^{\vee} \rightarrow E_{D_{i}}^{\bullet}$ and $\left[W_{j} \rightarrow M_{j}\right]^{\vee} \rightarrow E_{G_{j}}^{\bullet}$ which we then denote by $F_{D_{i}}^{\bullet}$ and $F_{G_{j}}^{\bullet}$.

An explicit quasi-isomorphism for the dual mapping cones is given by the diagram

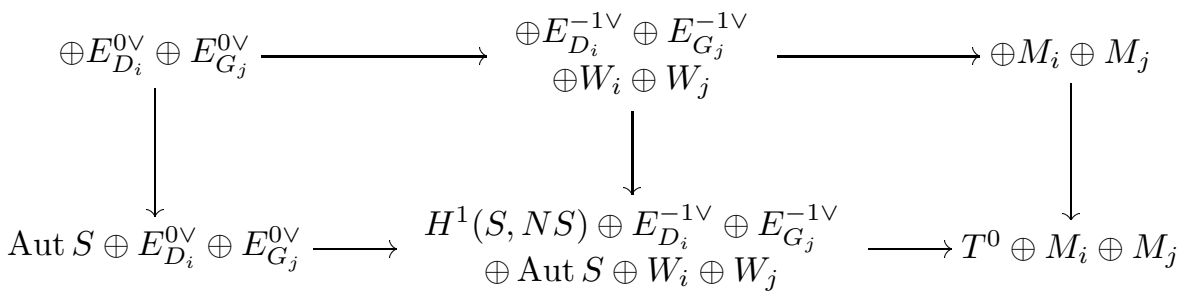

where the downward arrows are inclusions. Here we have chosen representatives of the objects $E_{S}, E_{D_{i}}$, and $E_{G_{j}}$ by 2-term complexes of sheaves (this can be done, e.g. as in [5]). The above diagram commutes because the only off diagonal map is the one from Aut $S \rightarrow \oplus M_{i} \oplus M_{j}$ on the bottom row. The fact that the diagram is a quasi-isomorphism follows because the maps on the bottom row are the identity restricted to Aut $S \rightarrow$ Aut $S$ and an isomorphism on $H^{1}(S, N S) \rightarrow T^{0}$.

To see this last isomorphism, we first note that there is an identification of $T^{0}:=$ $T_{t_{0}} T$ with $H^{2}\left(X, \mathcal{O}_{X}\right)$ as follows. For any $K 3$ surface $X$ the pairing $\Omega_{X}^{2} \otimes T_{X} \rightarrow \Omega_{X}^{1}$ induces a non-degenerate pairing

$$
H^{0}(X, K) \otimes H^{1}\left(X, T_{X}\right) \rightarrow H^{1}\left(X, \Omega_{X}^{1}\right) .
$$

The deformation theoretic interpretation of this pairing is that a holomorphic 2form $\Omega$ is deformed under an infinitesimal deformation $t \in H^{1}\left(X, T_{X}\right)$ in the $H^{1,1}(X)$ direction given by the image of $\Omega \otimes t$ under the above pairing. In the case of the twistor family $T$ determined by the Kähler structure $\omega \in H^{1}\left(X, \Omega_{X}^{1}\right)$, the infinitesimal change in a holomorphic 2 -form under a twistor deformation is by definition the Kähler form $\omega$. Thus the above pairing restricts to the isomorphism

$$
H^{0}(X, K) \otimes T^{0} \rightarrow \omega \mathbf{C}
$$

and so we see that $T^{0} \cong H^{0}(X, K)^{\vee} \cong H^{2}\left(X, \mathcal{O}_{X}\right)$.

One can then examine the deformation theory of the triple $S \subset X \subset Z$ (recall that $Z$ is the total space of the twistor family) to see that the map $H^{1}(S, N S) \rightarrow$ $T^{0} \cong H^{2}(X, \mathcal{O})$ is in fact given by the connecting homomorphism in the long exact cohomology sequence associated to

$$
0 \rightarrow \mathcal{O}_{X} \rightarrow \mathcal{O}_{X}(S) \rightarrow \mathcal{O}_{S}(S) \rightarrow 0
$$

It follows that the map $H^{1}(S, N S) \rightarrow H^{2}(X, \mathcal{O})$ is an isomorphism since $H^{1}(X, \mathcal{O}(S))$ and $H^{2}(X, \mathcal{O}(S))$ are 0 . 
Lemma 5.4 along with Proposition 7.4 of [6] shows that

$$
\left[\overline{\mathcal{M}}_{s, \Lambda}\right]^{v i r}=\left[\overline{\mathcal{M}}_{s, \Lambda}, F^{\bullet}\right]=\prod_{i}\left[\overline{\mathcal{M}}_{s\left(a_{i}\right)}, F_{D_{i}}^{\bullet}\right] \prod_{j}\left[\overline{\mathcal{M}}_{\Lambda\left(b_{j}\right)}, F_{G_{j}}^{\bullet}\right]
$$

We next describe the obstruction theories $F_{D_{i}}^{\bullet}$ and $F_{G_{j}}^{\bullet}$ in a more useful way. Note that in the construction of $F_{D_{i}}^{\bullet}$ and $F_{G_{j}}^{\bullet}$ in Lemma [5.4, we assumed that all the $a_{i}$ 's and $b_{j}$ 's were non-zero for notational convenience only. The same construction applies to the case where $a_{i}=0$ for all $i \neq i_{0}$ and $g=08$ In this case the moduli space $\overline{\mathcal{M}}_{s, \Lambda}$ is just $\overline{\mathcal{M}}_{s\left(a_{i_{0}}\right)}$ and thus its virtual class is given by $F_{D_{i_{0}}}^{\bullet}$. Similarly, if $a_{i}=0$ for all $i$ and $g=1$, then $\overline{\mathcal{M}}_{s, \Lambda}=\overline{\mathcal{M}}_{\Lambda(b)}$ and its virtual class is given by $F_{G_{j}}^{\bullet}$. In this case, $\overline{\mathcal{M}}_{\Lambda(b)}$ is just a single reduced point (with a trivial automorphism group) and so its virtual class $\left[\overline{\mathcal{M}}_{\Lambda(b)}, F_{G}^{\bullet}\right]=1$. Thus equation (7) reduces to

$$
\left[\overline{\mathcal{M}}_{s, \Lambda}\right]=\prod_{i}\left[\overline{\mathcal{M}}_{s\left(a_{i}\right)}, F_{s\left(a_{i}\right)}^{\bullet}\right]
$$

where we have changed notation from $F_{D_{i}}^{\bullet}$ to the slightly more descriptive $F_{s\left(a_{i}\right)}^{\bullet}$.

As we argued in the previous subsection, all the maps in $\overline{\mathcal{M}}_{s(a)}$ factor through the fixed local embedding $h: \Sigma(a) \rightarrow X$. By definition $\overline{\mathcal{M}}_{s(a)} \cong \overline{\mathcal{M}}_{0,0}(\Sigma(a), s(a))$ (recall that the sequence $s(a)=\left\{\ldots, s_{-1}(a), s_{0}(a), s_{1}(a), \ldots\right\}$ determines the degree of the maps on the various components of $\Sigma(a))$. The obstruction theory $F_{s(a)}^{\bullet}$ is obtained (via the proof of Lemma [5.4) from the diagram:

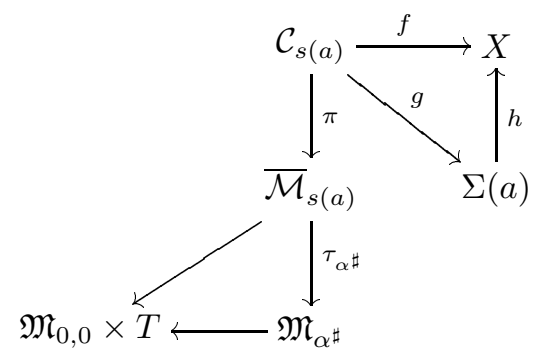

We see from the construction of $F_{s(a)}^{\bullet}$ that it does not depend on $X$, but only on the bundle $h^{*} T_{X}$ on $\Sigma(a)$. Furthermore, from the proof of Lemma 5.4 we see that the only role that the twistor family played in $F_{s(a)}^{\bullet(a)}$ was to cancel $H^{1}(S, T S)$, the obstruction to deforming $S$. In the above notation, this obstruction is $H^{1}\left(\Sigma_{*}, h^{*} T_{X}\right)$ (recall from the previous subsection that $\Sigma_{*} \subset \Sigma(a)$ is the component mapped isomorphically onto $S$ ). This discussion leads to the following characterization of $F_{s(a)}^{\bullet}$ :

Lemma 5.5. Let $T \rightarrow \Sigma(a)$ be a bundle on $\Sigma(a)$ such that $T$ is isomorphic to $h^{*} T_{X}$ restricted to the union of all the components of $\Sigma(a)$ except $\Sigma_{*}$ and $\left.T\right|_{\Sigma_{*}} \cong$

\footnotetext{
${ }^{8}$ The only real difference in this case that is worth noting is that the map $\mathfrak{M}_{\alpha \sharp} \rightarrow \mathfrak{M}_{0,0}$ is not a local embedding. It is in fact a composition of a quotient and a local embedding: $\mathfrak{M}_{\alpha^{\sharp}} \rightarrow$ $\mathfrak{M}_{\alpha^{\prime}} \hookrightarrow \mathfrak{M}_{0,0}$. Here $\alpha^{\prime}$ is the modular graph with 2 genus zero vertices and one edge connecting them. The first map is the quotient which forgets the fixed parameterization of the $S$ component. The fiber is the group of automorphisms of $S$ that fix the node. The second map is the usual local embedding defined by the modular graph $\alpha^{\prime}$. Thus the only modification required for this case is that we need to apply the two cases of Proposition A.1 in succession to get the analogue of the triangles in equation (5).
} 
$T \Sigma_{*} \oplus \mathcal{O}_{\Sigma_{*}}(-1)$. Then

$$
\left[R^{\bullet} \pi_{*} g^{*} T\right]^{\vee} \rightarrow L \overline{\overline{\mathcal{M}}}_{s(a)} / \mathfrak{M}_{0,0}
$$

defines a relative obstruction theory whose virtual class is isomorphic to $\left[\overline{\mathcal{M}}_{s(a)}, F_{s(a)}^{\bullet}\right]$.

Proof. Running through the same procedure as in the proof of Lemma 5.4 but using no twistor family and the bundle $T$ instead of $f^{*} T_{X} \otimes \mathcal{O}(-\mathbf{x})$, we encounter identical terms as we do for $F_{s(a)}^{\bullet}$, except the terms $H^{1}(S, N S)$ and $T^{0}$, which cancel in the quasi-isomorphism.

Remark 5.6. If the section $S \subset X$ had had self-intersection -1 to begin with, there would have been no need for the twistor family and all other arguments would have been the same. In particular, in the case of the rational elliptic surface, which is $\mathbf{C P}^{2}$ blown-up at nine points, the methods of this section apply to the ordinary Gromov-Witten invariants (i.e. no twistor family). This is carried out in Section 6

The results of this subsection can be summarized by saying that

$$
\left[\overline{\mathcal{M}}_{s, \Lambda}\right]^{v i r}=\prod_{i}\left[\overline{\mathcal{M}}_{s\left(a_{i}\right)}, F_{s\left(a_{i}\right)}^{\bullet}\right]
$$

and $F_{s\left(a_{i}\right)}^{\bullet}$ is characterized in Lemma 5.5 .

5.3. Computations via blow-ups on $\mathbf{P}^{2}$. In the previous subsection, we showed that the virtual fundamental cycle of $\overline{\mathcal{M}}_{s, \Lambda}$ is given by the product of virtual fundamental cycles on $\overline{\mathcal{M}}_{s\left(a_{i}\right)}$ defined by the obstruction theory $F_{s\left(a_{i}\right)}^{\bullet}$. In this subsection, we will realize the moduli space and its obstruction theory $\left(\overline{\mathcal{M}}_{s\left(a_{i}\right)}, F_{s\left(a_{i}\right)}^{\bullet}\right)$ as one coming from $\tilde{P}$, a certain blow-up of $\mathbf{P}^{2}$ at $2 a+3$ points.

The homology classes of $\tilde{P}$ will have a diagonal basis $h, e_{-a-1}, \ldots, e_{a+1}$ where $h^{2}=1$ and $e_{n}^{2}=-1$. We construct $\tilde{P}$ as follows. Begin with a linear $\mathbf{C}^{*}$ action on $\mathbf{P}^{2}$ fixing a line $H$ and a point $p$. Choose three points $p_{-}, p_{0}$, and $p_{+}$on $H$ and blow them up to obtain three exceptional curves $E_{-1}, E_{0}$, and $E_{1}$ representing classes $e_{-1}, e_{0}$, and $e_{1}$. The proper transform of $H$ is a $(-2)$-curve $\Sigma_{0}$ in the class $h-e_{-1}-e_{0}-e_{1}$. The $\mathbf{C}^{*}$ action extends to this blow-up acting with two fixed points on each of the curves $E_{-1}, E_{0}$, and $E_{1}$, namely the intersection with $\Sigma_{0}$ and one other. Blow-up the fixed points on $E_{-1}$ and $E_{1}$ that are not the ones on $\Sigma_{0}$ to obtain two new exceptional curves $E_{-2}$ and $E_{2}$ in the classes $e_{-2}$ and $e_{2}$. Let $\Sigma_{-1}$ and $\Sigma_{1}$ be the proper transforms of $E_{-1}$ and $E_{1}$ and note that they are (-2)spheres in the classes $e_{-1}-e_{-2}$ and $e_{1}-e_{2}$ respectively. The $\mathbf{C}^{*}$ action extends to this blow-up and we can repeat the procedure $a-1$ additional times to obtain $\tilde{P}$. $\tilde{P}$ contains $2 a+1(-2)$-spheres, namely $\Sigma_{-a}, \ldots, \Sigma_{a}$ which represent the classes

$$
\left[\Sigma_{n}\right]= \begin{cases}e_{n}-e_{n+1} & \text { if } 0<n \leq a, \\ h-e_{0}-e_{-1}-e_{1} & \text { if } a=0, \\ e_{n}-e_{n-1} & \text { if }-a \leq n<0 .\end{cases}
$$

We rename the (-1)-spheres $E_{0}, E_{a+1}$, and $E_{-a-1}$ by $\Sigma_{*}, \Sigma_{a+1}$, and $\Sigma_{-a-1}$ and it is a straightforward computation to check that the classes $\left[\Sigma_{*}\right],\left[\Sigma_{-a-1}\right], \ldots,\left[\Sigma_{a+1}\right]$ form an integral basis for $H_{2}(\tilde{P} ; \mathbf{Z})$. 


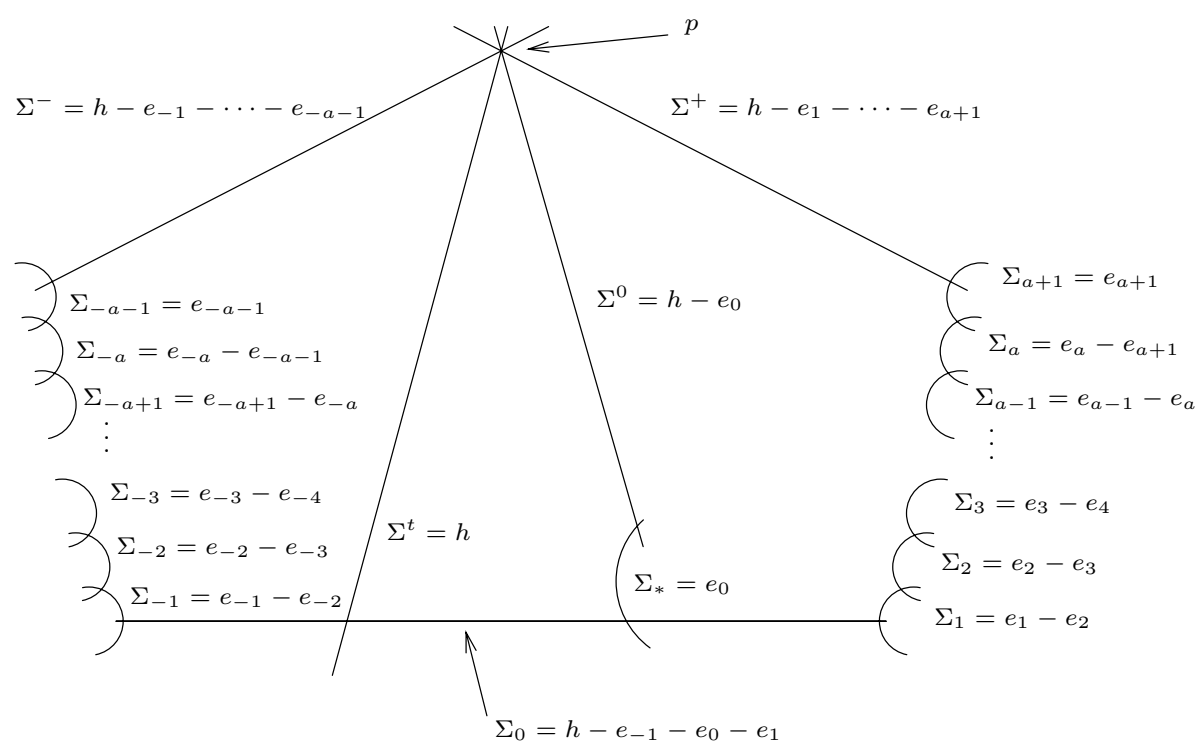

FiguRE 5.

The configuration $\Sigma_{*}+\sum_{n=-a}^{a} \Sigma_{n}$ is (as our notation suggests) biholomorphic to $\Sigma(a)$. Furthermore, $\left.T \tilde{P}\right|_{\Sigma(a)}$ is isomorphic to the bundle $T$ defining the obstruction theory $F_{s(a)}^{\bullet}$ (see Lemma[5.5). This will allow us to realize our obstruction problem as an ordinary Gromov-Witten invariant:

Lemma 5.7. $\left[\overline{\mathcal{M}}_{s(a)}\right]^{\text {vir }}:=\left[\overline{\mathcal{M}}_{s(a)}, F_{s(a)}^{\bullet}\right]^{\text {vir }}$ is the same as the (ordinary) genus 0 Gromov-Witten invariant of $\tilde{P}$ in the class

$$
\left[\Sigma_{*}\right]+\sum_{n=-a}^{a} s_{n}\left[\Sigma_{n}\right]
$$

Proof. This follows immediately from Lemma 5.5 if we can show that all the rational curves in the above homology class lie in the configuration $\Sigma(a)$.

Note that the curves $\Sigma_{*}, \Sigma_{-a-1}, \ldots, \Sigma_{a+1}$ are preserved by the $\mathbf{C}^{*}$ action and the only other curves preserved are the proper transforms of lines through the fixed point $p$. We call these additional lines $\Sigma^{+}, \Sigma^{-}, \Sigma^{0}$, and $\Sigma^{t}$ which are the proper transforms of the lines $\overline{p p_{+}}, \overline{p p_{-}}, \overline{p p_{0}}$, and $\overline{p p_{t}}$ where $p_{t}$ is any point on $H$ that is not $p_{+}, p_{-}$, or $p_{0}$ (see Figure 5 ).

We express the classes of $\Sigma^{+}, \Sigma^{-}, \Sigma^{0}$ and $\Sigma^{t}$ in the two homology bases $\left\{h, e_{-a-1}, \ldots, e_{a+1}\right\}$ and $\left\{\left[\Sigma_{*}\right],\left[\Sigma_{-a-1}\right], \ldots,\left[\Sigma_{a+1}\right]\right\}$ as follows:

$$
\begin{aligned}
& {\left[\Sigma^{t}\right]=h=\left[\Sigma_{*}\right]+\sum_{n=-a-1}^{a+1}\left[\Sigma_{n}\right],} \\
& {\left[\Sigma^{0}\right]=h-e_{0}=\sum_{n=-a-1}^{a+1}\left[\Sigma_{n}\right],}
\end{aligned}
$$




$$
\begin{aligned}
{\left[\Sigma^{+}\right] } & =h-e_{1}-\cdots-e_{a+1} \\
& =\left[\Sigma_{*}\right]+\sum_{n=-a-1}^{a+1}\left[\Sigma_{n}\right]-\sum_{n=1}^{a+1} n\left[\Sigma_{n}\right], \\
{\left[\Sigma^{-}\right] } & =h-e_{-1}-\cdots-e_{-a-1} \\
& =\left[\Sigma_{*}\right]+\sum_{n=-a-1}^{a+1}\left[\Sigma_{n}\right]-\sum_{n=1}^{a+1} n\left[\Sigma_{-n}\right] .
\end{aligned}
$$

Since $\mathbf{C}^{*}$ acts on $\tilde{P}$ we get a $\mathbf{C}^{*}$ action on the moduli space $\overline{\mathcal{M}}_{s(a)}^{\tilde{P}}$ of genus 0 stable maps to $\tilde{P}$ in the class $\left[\Sigma_{*}\right]+\sum_{n=-a}^{a} s_{n}\left[\Sigma_{n}\right]$. We first show that the maps in the fixed point set of $\overline{\mathcal{M}}_{s(a)}^{\tilde{P}}$ must have image $\Sigma_{*}+\sum_{n=-a}^{a} s_{n} \Sigma_{n}$. This is essentially for homological reasons: the image of a map in the fixed point set of $\overline{\mathcal{M}}_{s(a)}^{\tilde{P}}$ must be of the form

$$
c_{*} \Sigma_{*}+\sum_{n=-a-1}^{a+1} c_{n} \Sigma_{n}+\sum_{t} c^{t} \Sigma^{t}+c^{+} \Sigma^{+}+c^{-} \Sigma^{-}+c^{0} \Sigma^{0}
$$

for non-negative coefficients given by the $c$ 's. Since $\left[\Sigma_{*}\right],\left[\Sigma_{-a-1}\right], \ldots,\left[\Sigma_{a+1}\right]$ form a basis we have

$$
\begin{aligned}
c_{*}+\sum_{t} c^{t}+c^{+}+c^{-} & =1, \\
c_{n}+c^{0}+\sum_{t} c^{t}+c^{+}+c^{-}-|n| c^{\operatorname{sign}(n)} & = \begin{cases}s_{n}, & |n| \leq a, \\
0, & |n|=a+1 .\end{cases}
\end{aligned}
$$

The first equation implies that exactly one of $c_{*}, c^{t}, c^{+}$, or $c^{-}$is 1 (for some $t$ ) and the rest are 0 . Suppose that $c^{+}=1$; then $c^{-}=0$ and letting $n=-a-1$ in the second equation leads to a contradiction and so we have $c^{+}=0$. A similar argument shows $c^{-}=0$ and then summing the second equation over $n$ leads to

$$
\left(\sum_{n=-a-1}^{a+1} c_{n}\right)+(2 a+3)\left(c^{0}+\sum_{t} c^{t}\right)=a
$$

which implies that $c^{0}=c^{t}=0$. Thus $c_{*}=1$ and $c_{n}=s_{n}$.

Finally, suppose $f \in \overline{\mathcal{M}}_{s(a)}^{\tilde{P}}$ is not a fixed point of the $\mathbf{C}^{*}$ action. Then the limit of the action of $\lambda \in \mathbf{C}^{*}$ on $f$ as $\lambda \rightarrow 0$ must be fixed and hence has image $\Sigma_{*}+\sum_{n=-a}^{a} s_{n} \Sigma_{n}$. But then the limit of the action as $\lambda \rightarrow \infty$ must also be fixed and its image must contain the point $p$, which is a contradiction. Hence every $f \in \overline{\mathcal{M}}_{s(a)}^{\tilde{P}}$ is fixed by $\mathbf{C}^{*}$ and so has image $\Sigma_{*}+\sum_{n=-a}^{a} s_{n} \Sigma_{n}$.

Now $\tilde{P}$ is deformation equivalent to the blow-up of $\mathbf{P}^{2}$ at $2 a+3$ generic points and so the invariant for the class $\left[\Sigma_{*}\right]+\sum_{n=-a}^{a} s_{n}\left[\Sigma_{n}\right]$ can be computed using elementary properties of the invariants for blow-ups of $\mathbf{P}^{2}$.

We follow the notation of [20] and recall some of the properties of the invariant. We write $N\left(d ; \alpha_{1}, \ldots\right)$ for the genus 0 Gromov-Witten invariant in the class $d h-\sum_{i} \alpha_{i} e_{i}$. Here we are not being very picky about the indexing set for the exceptional classes since the invariant is the same under reordering. In the notation $N\left(d ; \alpha_{1}, \ldots\right)$ it is implicit that if the moduli space of genus 0 maps in the class 
$\left(d ; \alpha_{1}, \ldots\right)$ is positive dimensional, then we impose the proper number of point constraints and if the dimension is negative the invariant is zero. Also, we drop any $\alpha=0$ terms from the notation so that $N\left(d ; \alpha_{1}, \ldots, \alpha_{l}, 0, \ldots, 0\right)=N\left(d ; \alpha_{1}, \ldots, \alpha_{l}\right)$. The invariants satisfy the following properties:

1. $N\left(d ; \alpha_{1}, \ldots\right)=0$ if any $\alpha<0$ unless $d=0, \alpha_{i}=0$ for all $i$ except $i_{0}$ and $\alpha_{i_{0}}=-1$. In the latter case the invariant is 1 .

2. $N\left(d ; \alpha_{1}, \ldots, \alpha_{l}, 1\right)=N\left(d ; \alpha_{1}, \ldots, \alpha_{l}\right)$.

3. $N\left(d ; \alpha_{1}, \ldots, \alpha_{l}\right)=N\left(d ; \alpha_{\sigma(1)}, \ldots, \alpha_{\sigma(l)}\right)$ for any permutation $\sigma$.

4. $N\left(d ; \alpha_{1}, \ldots, \alpha_{l}\right)$ is invariant under the Cremona transformation which takes the class

$$
\left(d ; \alpha_{1}, \alpha_{2}, \alpha_{3}, \ldots\right)
$$

to the class

$$
\left(2 d-\alpha_{1}-\alpha_{2}-\alpha_{3} ; d-\alpha_{2}-\alpha_{3}, d-\alpha_{1}-\alpha_{3}, d-\alpha_{1}-\alpha_{2}, \ldots\right) .
$$

5. $N(1)=1$.

Ordering the exceptional classes in $\tilde{P}$ by $e_{0}, e_{1}, e_{-1}, e_{2}, e_{-2}, \ldots$ and rewriting the class $\Sigma_{*}+\sum s_{n} \Sigma_{n}$ in this basis, we can express the contribution of $\overline{\mathcal{M}}_{s(a)}$ as

$$
\left[\overline{\mathcal{M}}_{s(a)}\right]^{v i r}=N\left(s_{0} ; s_{0}-1, s_{0}-s_{1}, s_{0}-s_{-1}, s_{1}-s_{2}, s_{-1}-s_{-2}, \ldots, s_{-a+1}-s_{-a}\right) .
$$

We call an admissible sequence $\left\{s_{n}\right\}$ 1-admissible if $s_{ \pm n \pm 1}$ is either $s_{ \pm n}$ or $s_{ \pm n}-1$ for all non-negative $n$.

Lemma 5.8. $\left[\overline{\mathcal{M}}_{s(a)}\right]^{\text {vir }}=1$ if $s(a)$ is a 1-admissible sequence and $\left[\overline{\mathcal{M}}_{s(a)}\right]^{\text {vir }}=0$ otherwise.

Proof. Suppose that $\left[\overline{\mathcal{M}}_{s(a)}\right]^{v i r} \neq 0$. Since $s_{0}>0$, all the other terms in $\left(s_{0} ; s_{0}-\right.$ $\left.1, s_{0}-s_{1}, \ldots\right)$ must be non-negative by property 1 . Thus $s_{ \pm n \pm 1} \leq s_{ \pm n}$ for all $n$. Now by permuting and performing the Cremona transformation, we get

$$
\begin{aligned}
{\left[\overline{\mathcal{M}}_{s(a)}\right]^{v i r}=} & N\left(s_{0} ; s_{0}-1, s_{0}-s_{1}, s_{ \pm n}-s_{ \pm n \pm 1}, \ldots\right) \\
= & N\left(1+s_{1}+s_{ \pm n \pm 1}-s_{ \pm n} ; s_{1}-s_{ \pm n}+s_{ \pm n \pm 1},\right. \\
& \left.1+s_{ \pm n \pm 1}-s_{ \pm n}, s_{1}+1-s_{0}, \ldots\right) .
\end{aligned}
$$

Now since $s_{ \pm n} \leq s_{1}$, we have $1+s_{1}+s_{ \pm n \pm 1}-s_{ \pm n}>0$ and so $1+s_{ \pm n \pm 1}-s_{ \pm n} \geq 0$ which combined with $s_{ \pm n \pm 1} \leq s_{ \pm n}$ yields

$$
s_{ \pm n \pm 1} \leq s_{ \pm n} \leq s_{ \pm n \pm 1}+1,
$$

and so $s$ is 1-admissible.

Suppose then that $s$ is 1-admissible. Then except for the first two terms, the class $\left(s_{0} ; s_{0}-1, s_{0}-s_{1}, \ldots\right)$ consists of 0 's and 1's. Thus $\left[\overline{\mathcal{M}}_{s(a)}\right]^{\text {vir }}=N\left(s_{0} ; s_{0}-1\right)$. Finally, since $N\left(s_{0} ; s_{0}-1\right)=N\left(s_{0} ; s_{0}-1,1,1\right)$ we can apply Cremona to get

$$
N\left(s_{0} ; s_{0}-1\right)=N\left(s_{0}-1 ; s_{0}-2\right)
$$

and so by induction

$$
\left[\overline{\mathcal{M}}_{s(a)}\right]^{v i r}=N\left(s_{0} ; s_{0}-1\right)=N(1)=1
$$

and the lemma is proved.

Lemma 5.9. The number of 1-admissible sequences $s$ with $|s|=a$ is the number of partitions of $a, p(a)$. 
Proof. 9 The number of partitions, $p(a)$, is given by the number of Young diagrams of size $a$. There is a bijective correspondence between 1-admissible sequences and Young diagrams. Given a Young diagram define a 1-admissible sequence $\left\{s_{n}\right\}$ by setting $s_{0}$ equal to the number of blocks on the diagonal, $s_{1}$ equal to the number of blocks on the first lower diagonal, $s_{2}$ equal to the number of blocks on the second lower diagonal, and so on, doing the same for $s_{-1}, s_{-2}, \ldots$ with the upper diagonals. It is easily seen that this defines a bijection.

Summarizing the results of this section we have:

Theorem 5.10. Since every component of $\overline{\mathcal{M}}_{\mathbf{a}, \mathbf{b}}$ contributes either 0 or 1 to $N_{g}(n)$, the overall contribution of $\overline{\mathcal{M}}_{\mathbf{a}, \mathbf{b}}$ is the sum over all the connected components whose contribution is 1 . It is thus the sum of all choices of data $(s(\mathbf{a}), \Lambda(\mathbf{b}))$ such that all the sequences of $s(\mathbf{a})$ are 1-admissible. For each $a_{j} \in \mathbf{a}$ we have $p\left(a_{j}\right)$ choices of a 1-admissible sequence $s\left(a_{j}\right)$ and for each $b_{i} \in \mathbf{b}$ we have $b_{j} \sigma\left(b_{i}\right)$ choices for the data $\Lambda\left(b_{i}\right)$. Thus the total contribution is:

$$
\left[\overline{\mathcal{M}}_{\mathbf{a}, \mathbf{b}}\right]^{v i r}=\prod_{j=1}^{24} p\left(a_{j}\right) \prod_{i=1}^{g} b_{i} \sigma\left(b_{i}\right) .
$$

\section{Counting Curves on the Rational elliptic Surface}

Let $Y$ be the blow-up of $\mathbf{P}^{2}$ at nine distinct points. In this section we apply our degeneration method and our local calculations to compute a certain set of Gromov-Witten invariants of $Y$. We compute the genus $g$ invariants for all classes such that the invariants require exactly $g$ constraints. There is a canonical symplectic form $\omega$ (unique up to deformation equivalence) on $Y$ determined by the blow up of the Fubini-Study form on $\mathbf{P}^{2}$.

If we arrange these nine blow-up points lying on a pencil of cubic elliptic curves in $\mathbf{P}^{2}$, then $Y$ has the structure of an elliptic surface with fiber class $F$ representing these elliptic curves in $H_{2}(Y, \mathbf{Z})$ and the nine exceptional curves $e_{1}, e_{2}, \ldots, e_{9}$ are all sections of this elliptic fibration. If $h$ represents the homology class of the strict transform of the hyperplane in $\mathbf{P}^{2}$, then we have $F=3 h-e_{1}-\cdots-e_{9}$. In fact $H^{2}(Y, \mathbf{Z})$ is generated by $e_{1}, \ldots, e_{9}$ and $h$. We abbreviate the class $d h-a_{1} e_{1}-\cdots-$ $a_{9} e_{9}$ by $\left(d ; a_{1}, \ldots, a_{9}\right)$.

Now we pick any of these sections, $e_{9}$ say, and consider the class $C_{n}=e_{9}+$ $(g+n) F=(3(n+g) ; g+n, \ldots, g+n, g+n-1)$. It is easy to check that the complete linear system $\left|C_{n}\right|$ has dimension $g+n$. We write $N_{g}^{Y}(C)$ for the GromovWitten invariant for $(Y, \omega)$ which counts the number of curves of geometric genus $g$ representing the homology class $C$ and passing through $g$ points, i.e. we define

$$
N_{g}^{Y}(C)=\Psi_{(C, g, g)}^{Y}\left(\left[p_{1}\right], \ldots,\left[p_{g}\right]^{\vee}\right) .
$$

We show that the numbers $N_{g}^{Y}\left(C_{n}\right)$ contain all the genus $g$ Gromov-Witten invariants that are constrained to exactly $g$ points. This was observed by Göttsche who explained the following argument to us:

For $N_{g}^{Y}(C)$ to be well defined (see Equation (11)) we need $4 g=2 c_{1}(Y) \cdot C+2 g-$ $2(1-g)$, i.e. $F \cdot C=1$. Now the Gromov-Witten invariants do not change when $C \mapsto C^{\prime}$ is induced by a permutation of the exceptional classes $e_{i}$ or a Cremona

\footnotetext{
${ }^{9}$ We are grateful to D. Maclagan and S. Schleimer for help with this and other combinatorial difficulties.
} 
transform (see [20]). Recall that the Cremona transform takes a class $\left(d ; a_{1}, \ldots, a_{9}\right)$ to the class

$$
\left(2 d-a_{1}-a_{2}-a_{3} ; d-a_{2}-a_{3}, d-a_{1}-a_{3}, d-a_{1}-a_{2}, a_{4}, \ldots, a_{9}\right) .
$$

Lemma 6.1. Let $C \in H_{2}(Y ; \mathbf{Z})$ be a class so that the moduli space of genus $g$ maps has formal dimension $g$. Then the class $C$ can be transformed by a sequence of Cremona transforms and permutations of the $e_{i}$ 's to a class of the form $e_{9}+$ $(g+n) F=C_{n}$.

Proof. By permuting the $E_{i}$ 's we may assume that $a_{1} \geq a_{2} \geq \cdots \geq a_{9}$. Then the condition $F \cdot C=1$ is equivalent to $3 d-1=\sum_{i} a_{i}$ so that $a_{1}+a_{2}+a_{3} \geq d$ with equality if and only if $C=(3 i, i, i, i, i, i, i, i, i, i-1)=e_{9}+i F$ for some $i=n+g$. If the equality is strict, then we can apply a Cremona transform to obtain $C^{\prime}=\left(e, b_{1}, \ldots, b_{9}\right)$ with $e<d$. The result follows by descending induction on $d$.

The methods of sections 4 and 5 apply to these invariants (see Remark 5.6). Note that the elliptic fibration of $Y$ has (generically) 12 nodal fibers rather than 24 . We get essentially the same formula as in the $K 3$ case with the 24 replaced by 12.

Theorem 6.2. For any $g \geq 0$, we have

$$
\begin{aligned}
\sum_{n=0}^{\infty} N_{g}^{Y}\left(C_{n}\right) q^{C_{n}^{2} / 2} & =\left(\sum_{b=1}^{\infty} b \sigma(b) q^{b}\right)^{g} q^{1 / 2} \prod_{m=1}^{\infty}\left(1-q^{m}\right)^{-12} \\
& =\frac{\left(D G_{2}\right)^{g}}{\sqrt{\Delta}} .
\end{aligned}
$$

When the genus $g$ equals zero, these numbers are computed by Göttsche and Pandharipande 20]. In fact, they obtain all genus zero Gromov-Witten invariants for $\mathbf{P}^{2}$ blown up at an arbitrary number of points in terms of two rather complicated recursive formulas. Theorem 6.2 can be verified term by term for $g=0$ using the recurrence relations, although the computer calculation becomes extremely lengthy quickly. We know of no way of obtaining the genus 0 closed form of Theorem 6.2 directly from the recurrence relations.

\section{Appendix A. Virtual Classes, point constraints, And Base Changes}

This appendix is concerned with proving some results about virtual classes 10 First we prove a general result about comparing the virtual classes given by perfect relative obstruction theories related by a base change. Second we prove a result well known to experts [4] but not present in the literature. Roughly, it says that, at least for point constraints, one can compute the Gromov-Witten invariants by either evaluating cohomology classes on the virtual class of the whole moduli space of stable maps (this is the usual set-up), or by constructing a natural virtual cycle directly on the "cut down" moduli space of maps which hit the prescribed points. For this appendix we use the purely algebro-geometric notions of Gromov-Witten theory as defined by Behrend-Fantechi ([6], [5]) and accordingly we will adjust our notation to more closely match the algebraic geometry literature.

\footnotetext{
10 We would like to thank Tom Graber for his invaluable assistance in these matters.
} 
A.1. Base changes for perfect relative obstruction theories. Let $\sigma: X \rightarrow Y$ be a morphism of stacks, and assume that $Y$ is smooth and $X$ is of Deligne-Mumford type. Let $f: Y \rightarrow Z$ be a morphism, and let $\tau: X \rightarrow Z$ be the composition:

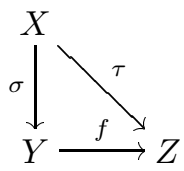

Proposition A.1. (1) Suppose that $f$ is smooth and that $F^{\bullet} \rightarrow L_{\sigma}^{\bullet}$ is a perfect relative obstruction theory (cf. [6]). Then there exists a perfect relative obstruction theory $E^{\bullet} \rightarrow L_{\tau}^{\bullet}$ fitting into the following morphism of triangles:

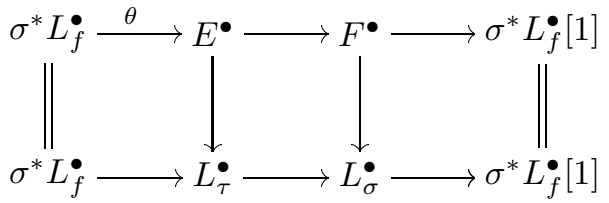

such that the virtual classes $\left[X, E^{\bullet}\right]$ and $\left[X, F^{\bullet}\right]$ coincide.

(2) Suppose that $f$ is a local embedding with $X$ and $Y$ smooth, and suppose that $E^{\bullet} \rightarrow L_{\tau}^{\bullet}$ is a perfect relative obstruction theory. If the map $h^{-1}\left(\sigma^{*} L_{f}^{\bullet}\right) \rightarrow h^{-1}\left(L_{\tau}^{\bullet}\right)$ factors through a map

$$
h^{-1}\left(\sigma^{*} L_{f}^{\bullet} \stackrel{\theta}{\longrightarrow} h^{-1}\left(E^{\bullet}\right) \longrightarrow h^{-1}\left(L_{\tau}^{\bullet}\right)\right.
$$

then the previous diagram exists and $F^{\bullet}$ is a perfect relative obstruction theory such that the virtual classes $\left[X, E^{\bullet}\right]$ and $\left[X, F^{\bullet}\right]$ coincide.

Remark A.2. In the case that $Z$ is a point, the proposition shows how to convert a perfect relative obstruction theory into an equivalent non-relative theory. This was employed in Gromov-Witten theory in [21], Appendix B.

Remark A.3. The case we are primarily interested in for this paper is when $f$ : $Y \rightarrow Z$ is a smooth local embedding. In Gromov-Witten theory, this arises if for a priori reasons it is known that the moduli space of stable maps consists of maps whose domains have some specified degeneration type $\rho^{\sharp}$. Then the usual relative obstruction theory for $\tau: \overline{\mathcal{M}}(X) \rightarrow \mathfrak{M}$ can be converted to a perfect relative obstruction theory for $\sigma: \overline{\mathcal{M}}(X) \rightarrow \mathfrak{M}_{\rho^{\sharp}}$ where $\mathfrak{M}_{\rho^{\sharp}} \subset \mathfrak{M}$ is the substack of the moduli stack of prestable curves that parameterizes curves of degeneration type $\rho^{\sharp}$. For the existence of the map $\theta$ in this case, see Corollary B.2 of Appendix B.

Proof of Proposition A.1 In general, an obstruction theory does not depend on the whole cotangent complex but only on the cut-off complex $\tau_{\geq-1} L^{\bullet}$ and so we can assume without loss of generality that $L_{\tau}^{\bullet}$ and $L_{\sigma}^{\bullet}$ have no cohomology in degrees less than -1 . The condition that $E^{\bullet}$ (resp. $F^{\bullet}$ ) is an obstruction theory implies that the mapping cone of $E^{\bullet} \rightarrow L_{\tau}$ (resp. $F^{\bullet} \rightarrow L_{\sigma}^{\bullet}$ ) has cohomology only in degree -2 . If the diagram in the theorem exists, then it can be completed to the diagram 
(see Weibel [35], page 378)

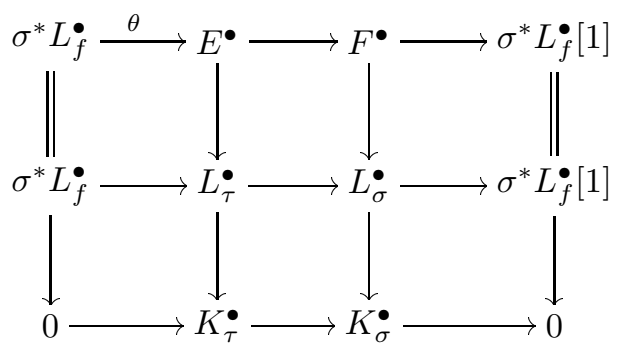

where the bottom row is an exact triangle and $K_{\tau}^{\bullet}$ and $K_{\sigma}^{\bullet}$ are the mapping cones of the vertical morphisms above them. This diagram implies that $K_{\tau}^{\bullet} \cong K_{\sigma}^{\bullet}$ and so if we know that $E^{\bullet}$ (resp. $F^{\bullet}$ ) is a perfect obstruction theory, then $F^{\bullet}$ (resp. $\left.E^{\bullet}\right)$ must satisfy the cohomological conditions of an obstruction theory. In order to then conclude that $F^{\bullet}$ (resp. $E^{\bullet}$ ) is a perfect obstruction theory in the sense of Behrend and Fantechi, we would need to prove that it has a global resolution by a two-term complex of vector bundles. This technical condition is no longer necessary due to recent work of Kresch (24], Section 6.2), so we just need the existence of the diagram in order to show that $F^{\bullet}$ (resp. $E^{\bullet}$ ) is an obstruction theory. In case (1), the diagram automatically exists by the mapping cone construction on the composite morphism $F^{\bullet} \rightarrow L_{\sigma}^{\bullet} \rightarrow \sigma^{*} L_{f}^{\bullet}[1]$.

For case (2), the morphism $\theta$ (and hence the rest of the diagram) exists if and only if the composition $\sigma^{*} L_{f}^{\bullet} \rightarrow L_{\tau}^{\bullet} \rightarrow K_{\tau}^{\bullet}$ is $0 . \sigma^{*} L_{f}^{\bullet}$ is supported in degree -1 since $f$ is a smooth local embedding. Now since $\sigma^{*} L_{f}^{\bullet}$ and $K^{\bullet}$ have cohomology in one degree only, they are determined in the derived category by their cohomology ([22], Proposition 4.3); however, since they have cohomology in different degrees, it is not the case that a morphism between them is determined by the induced map on cohomology. The additional hypothesis in the theorem is needed. It provides an obvious chain homotopy to 0 of the map of complexes (in degree $[-2,-1]$ ):

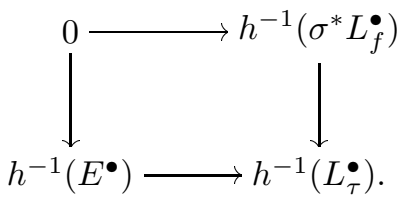

The above map of complexes represents the morphism $\sigma^{*} L_{f}^{\bullet} \rightarrow K_{\tau}^{\bullet}$ and so it is 0 in the derived category and thus $\theta$ exists.

To finish the proof of the proposition, we need to show that given the existence of the diagram in the theorem, the virtual classes $\left[X, E^{\bullet}\right]$ and $\left[X, F^{\bullet}\right]$ coincide. By definition, $\left[X, E^{\bullet}\right]\left(\right.$ resp. $\left.\left[X, F^{\bullet}\right]\right)$ is the intersection of the relative intrinsic normal cone stack $\mathfrak{C}_{X / Z}$ (resp. $\mathfrak{C}_{X / Y}$ ) with the 0 section of the vector bundle stack $\mathfrak{E}$ (resp. $\mathfrak{F}$ ). If $E^{\bullet}$ and $F^{\bullet}$ have global resolutions, then $\mathfrak{C}_{X / Z} \subset \mathfrak{E}$ and $\mathfrak{C}_{X / Y} \subset \mathfrak{F}$ induce cones $C_{X / Z} \subset E_{1}$ and $C_{X / Y} \subset F_{1}$ (here $E_{1}:=E^{-1 \vee}$ and $F_{1}:=F^{-1 \vee}$ ). We can then write

$$
\begin{aligned}
& {\left[X, E^{\bullet}\right]=0_{E_{1}}^{!}\left[C_{X / Z}\right],} \\
& {\left[X, F^{\bullet}\right]=0_{F_{1}}^{!}\left[C_{X / Y}\right] .}
\end{aligned}
$$


Then the injection $i: F_{1} \rightarrow E_{1}$ fits into the diagram of fiber squares:

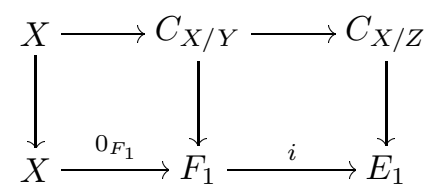

with $i \circ 0_{F_{1}}=0_{E_{1}}$. Thus we have

$$
\begin{aligned}
{\left[X, E^{\bullet}\right] } & =0_{E_{1}}^{!}\left[C_{X / Z}\right] \\
& =0 !{ }_{F_{1}} i^{!}\left[C_{X / Z}\right] \\
& =0 !{ }_{F_{1}}\left[C_{X / Y}\right] \\
& =\left[X, F^{\bullet}\right] .
\end{aligned}
$$

The fact that we assumed global resolutions is not really a restriction since Kresch's intersection theory for Artin stacks satisfies the same formal properties as ordinary intersection theory.

A.2. Virtual classes and point constraints. Let $X$ be a smooth projective variety, let $\beta \in H_{2}(X, \mathbf{Z})$, and let

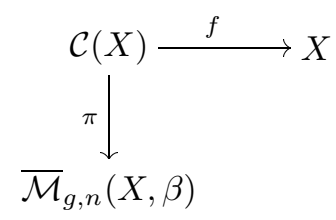

be the universal diagram for the moduli stack of genus $g, n$-marked stable maps in the class $\beta$. In the sequel we will drop the $\beta$ from the notation. The virtual class $\left[\overline{\mathcal{M}}_{g, n}(X)\right]^{v i r}$ is determined by a perfect relative obstruction theory which is a morphism

$$
\left[R^{\bullet} \pi_{*} f^{*} T_{X}\right]^{\vee} \rightarrow L_{\overline{\mathcal{M}}_{g, n}(X) / \mathfrak{M}_{g, n}}
$$

in the derived category of $\mathcal{O}_{\overline{\mathcal{M}}_{g, n}(X)}$ modules and $L_{\overline{\mathcal{M}}_{g, n}(X) / \mathfrak{M}_{g, n}}$ is the relative cotangent complex of $\overline{\mathcal{M}}_{g, n}(X)$ over $\mathfrak{M}_{g, n}$, the Artin stack of prestable genus $g$, $n$-marked curves.

There are $n$ universal sections of the universal family $x_{i}: \overline{\mathcal{M}}_{g, n}(X) \rightarrow \mathcal{C}(X)$ given by the marked points. Let

$$
e: \overline{\mathcal{M}}_{g, n}(X) \rightarrow X^{n}
$$

be the evaluation map defined by $e=\left(f \circ x_{1}\right) \otimes \cdots \otimes\left(f \circ x_{n}\right)$ and choose $n$ generic points $p_{1}, \ldots, p_{n} \in X$. Define the substack $\overline{\mathcal{M}}_{g, n}(X, \mathbf{p}) \subset \overline{\mathcal{M}}_{g, n}(X)$ by the Cartesian square

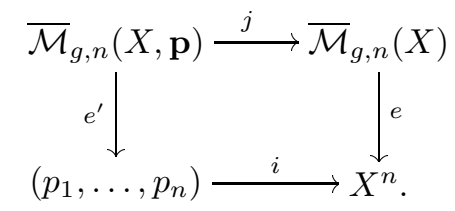


$\overline{\mathcal{M}}_{g, n}(X, \mathbf{p})$ is the cut down moduli stack parameterizing those maps where the $i$ th marked point maps to $p_{i}$. We will abuse notation by using $x_{i}$ to refer to the map and the divisor given by $\operatorname{Im}\left(x_{i}\right)$.

Proposition A.4. There exists a relative perfect obstruction theory

$$
j^{*}\left[R^{\bullet} \pi_{*}\left(f^{*} T_{X}\left(-x_{1}-\cdots-x_{n}\right)\right)\right]^{\vee} \rightarrow L_{\overline{\mathcal{M}}_{g, n}(X, \mathbf{p}) / \mathfrak{M}_{g, n}}
$$

defining a virtual class $\left[\overline{\mathcal{M}}_{g, n}(X, \mathbf{p})\right]^{\text {vir }}$ so that

$$
\left[\overline{\mathcal{M}}_{g, n}(X, \mathbf{p})\right]^{\text {vir }}=e^{*}\left(\mathbf{p}^{\vee}\right) \cap\left[\overline{\mathcal{M}}_{g, n}(X)\right]^{\text {vir }}
$$

where $\mathbf{p}^{\vee}$ is the class Poincare dual to a point in $X^{n}$. Note that the right hand side is what appears in the usual definition of Gromov-Witten invariants (for point constraints). The formula can be written equivalently as

$$
\left[\overline{\mathcal{M}}_{g, n}(X, \mathbf{p})\right]^{v i r}=i^{!}\left[\overline{\mathcal{M}}_{g, n}(X)\right]^{v i r} .
$$

Proof. We write $\mathcal{O}(-\mathbf{x})$ to denote $\mathcal{O}\left(-x_{1}-\cdots-x_{n}\right)$ and we will drop the $g$ and $n$ from the notation for the moduli stacks. Tensoring the divisor sequence

$$
0 \rightarrow \mathcal{O}(-\mathbf{x}) \rightarrow \mathcal{O} \rightarrow \bigoplus_{i} x_{i *} \mathcal{O}_{\overline{\mathcal{M}}(X)} \rightarrow 0
$$

by $f^{*} T_{X}$ we get

$$
0 \rightarrow f^{*} T_{X}(-\mathbf{x}) \rightarrow f^{*} T_{X} \rightarrow \bigoplus_{i} x_{i *} \mathcal{O}_{\overline{\mathcal{M}}(X)} \otimes f^{*} T_{X} \rightarrow 0 .
$$

Note that

$$
\begin{gathered}
x_{i *} \mathcal{O}_{\overline{\mathcal{M}}(X)} \otimes f^{*} T_{X}=x_{i *}\left(x_{i}^{*} f^{*} T_{X}\right), \\
\bigoplus_{i} x_{i}^{*} f^{*} T_{X}=e^{*} T_{X^{n}},
\end{gathered}
$$

and $\pi \circ x_{i}=I d$, so by applying $R^{\bullet} \pi_{*}$ we get an exact triangle

$$
R^{\bullet} \pi_{*}\left(f^{*} T_{X}(-\mathbf{x})\right) \rightarrow R^{\bullet} \pi_{*} f^{*} T_{X} \rightarrow e^{*} T_{X^{n}} \rightarrow R^{\bullet} \pi_{*}\left(f^{*} T_{X}(-\mathbf{x})\right)[1] .
$$

Dualizing and pulling back by $j$ we get the triangle:

$$
j^{*}\left[R^{\bullet} \pi_{*} f^{*} T_{X}\right]^{\vee} \rightarrow j^{*}\left[R^{\bullet} \pi_{*}\left(f^{*} T_{X}(-\mathbf{x})\right)\right]^{\vee} \rightarrow j^{*} e^{*}\left(\Omega_{X^{n}}\right)[1] \rightarrow j^{*}\left[R^{\bullet} \pi_{*} f^{*} T_{X}\right]^{\vee}[1] .
$$

Furthermore, we have $j^{*} e^{*} \Omega_{X^{n}}[1] \cong e^{* *} i^{*} L_{X^{n}}^{\bullet}[1] \cong e^{* *} L_{\mathbf{p} / X^{n}}^{\bullet}$. Then using the isomorphism

$$
e^{\prime *} L_{\mathbf{p} / X^{n}} \rightarrow L_{\overline{\mathcal{M}}(X, \mathbf{p}) / \overline{\mathcal{M}}(X)}^{\bullet}
$$

and the perfect relative obstruction theory for $\overline{\mathcal{M}}(X) / \mathfrak{M}$ we can complete the following diagram to a morphism of triangles:

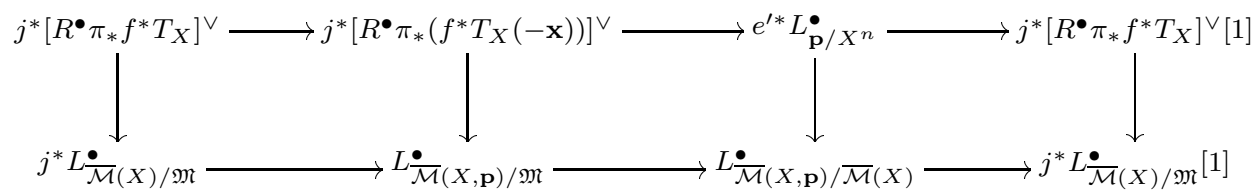

By the above diagram, the relative obstruction theories

$$
\left[R^{\bullet} \pi_{*} f^{*} T_{X}\right]^{\vee} \rightarrow L_{\overline{\mathcal{M}}(X) / \mathfrak{M}}
$$


and

$$
\left[R^{\bullet} \pi_{*}\left(f^{*} T_{X}(-\mathbf{x})\right)\right]^{\vee} \rightarrow L_{\overline{\mathcal{M}}(X, \mathbf{p}) / \mathfrak{M}}
$$

are compatible in the sense of Behrend and Fantechi (cf. page 86 in [6]). Since $i: \mathbf{p} \rightarrow X^{n}$ is a smooth embedding, Proposition 7.5 in [6] gives

$$
i^{!}[\overline{\mathcal{M}}(X)]^{v i r}=[\overline{\mathcal{M}}(X, \mathbf{p})]^{\text {vir }},
$$

which proves the proposition.

Remark A.5. For arbitrary cycles $\Gamma_{1}, \ldots, \Gamma_{n}$ one can form the analogous square to (8) and the substack $\overline{\mathcal{M}}(X, \Gamma)$. The perfect obstruction theory for $\overline{\mathcal{M}}(X, \Gamma)$ can then be obtained in a similar fashion as the mapping cone of the usual obstruction theory for $\overline{\mathcal{M}}(X)$ and $e^{* *} L_{\Gamma / X^{n}}^{\bullet}$.

\section{Appendix B. A Deformation Result}

In this appendix we prove the deformation result needed in subsection 5.2 (cf. Remark A.3). We use the notation of section 5 .

Fixing a point $(f: C \rightarrow X)$ in the moduli space $\overline{\mathcal{M}}(X, \mathbf{p}) / \mathfrak{M}_{g, g} \times T$, we have an exact sequence for $T^{1}$ and $T^{2}$ which are respectively the infinitesimal deformations of the map $f$ (as a stable map) and the obstructions to the deformations. The exact sequence is below:

$0 \rightarrow \operatorname{Aut} C \rightarrow H^{0}\left(C, f^{*} T_{X}(-\mathbf{x})\right) \rightarrow T^{1} \rightarrow \operatorname{Def} C \oplus T^{0} \rightarrow H^{1}\left(C, f^{*} T_{X}(-\mathbf{x})\right) \rightarrow T^{2}$ where (cf. subsection 5.2) $T^{0}:=T_{t_{0}} T$ is the space of infinitesimal deformations of $X$ in the direction of the twistor family.

Consider the diagram:

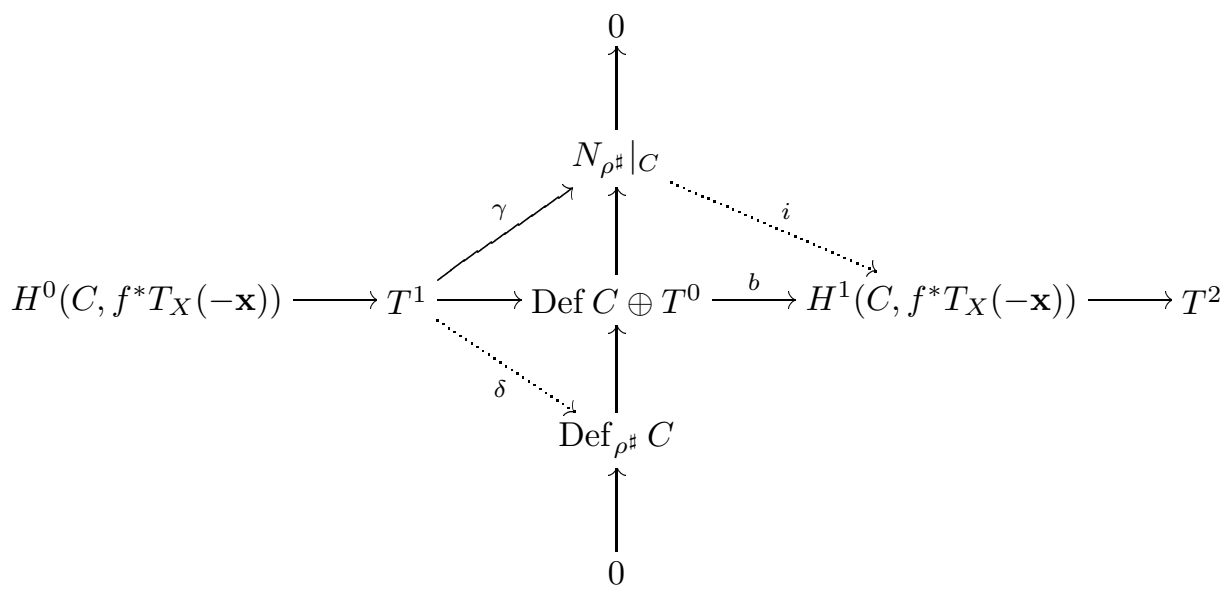

Here $\operatorname{Def}_{\rho^{\sharp}} C$ are deformations of $C$ preserving the type $\rho^{\sharp}$, i.e. preserving the $24+g$ nodes $y_{i}$ and $z_{j}$ and preserving the component $(S, \mathbf{y}, \mathbf{z})$ (see subsection 5.2 for notation). $\left.\quad N_{\rho^{\sharp}}\right|_{C}$ is the fiber over $C$ of the normal bundle of the embedding $\mathfrak{M}_{\rho^{\sharp}} \times t_{0} \hookrightarrow \mathfrak{M}_{g, g} \times T$.

Theorem B.1. The map $i$ in the above diagram exists and is split injective. Thus $\gamma=0$ and so the map $\delta$ exists; in other words, all infinitesimal deformations of $f: C \rightarrow X$ induce infinitesimal deformations of $C$ that land in $\operatorname{Def}_{\rho^{\sharp}} C$. 
Proof. We have an exact sequence for $H^{1}\left(C, f^{*} T_{X}(-\mathbf{x})\right)$ coming from the decomposition $C=S \cup_{i} D_{i} \cup_{j} G_{j}$ (cf. sequence (的)):

$$
\cdots \rightarrow \oplus T_{y_{i}} X \oplus T_{z_{j}} X \rightarrow H^{1}\left(C, f^{*} T_{X}(-\mathbf{x})\right) \rightarrow H^{1}\left(S, N_{S}\right) \stackrel{\oplus H^{1}\left(D_{i}, f^{*}\left(T_{X}\right)\right)}{\oplus H^{1}\left(G_{j}, f^{*} T_{X}\left(-x_{j}\right)\right)} \rightarrow 0 .
$$

There are splittings $T_{y_{i}} X=T_{y_{i}} S \oplus T_{y_{i}} N_{i}, T_{z_{j}} X=T_{z_{j}} S \oplus T_{z_{j}} F_{j}$ and the image of the previous term (i.e. $\left.H^{0}\left(S, T_{S}\right) \oplus H^{0}\left(D_{i}, f^{*} T_{X}\right)\right)$ is easy to identify. The $H^{0}\left(D_{i}, f^{*} T_{X}\right)$ factors map onto the $T_{y_{i}} N_{i}$ factors and $H^{0}\left(S, T_{S}\right)=$ Aut $S$ has its image in $\oplus T_{y_{i}} S \oplus T_{z_{j}} S$. Recall that $\operatorname{Def}(S, \mathbf{y}, \mathbf{z})=\oplus T_{y_{i}} S \oplus T_{z_{j}} S /$ Aut $S$ so we obtain the short exact sequence:

$$
0 \rightarrow \operatorname{Def}(S, \mathbf{y}, \mathbf{z}) \rightarrow H^{1}\left(C, f^{*} T_{X}(-\mathbf{x})\right) \rightarrow H^{1}\left(S, N_{S}\right) \underset{\oplus H^{1}\left(G_{j}, f^{*} T_{X}\left(-x_{j}\right)\right)}{\oplus H^{1}\left(D_{i} f^{*}\left(T_{X}\right)\right)} \rightarrow 0 .
$$

On the other hand we have an exact sequence for $\left.N_{\rho^{\sharp}}\right|_{C}$ (cf. subsection 5.2)

$$
0 \rightarrow \operatorname{Def}(S, \mathbf{y}, \mathbf{z}) \oplus T^{0} \rightarrow N_{\left.\rho^{\sharp}\right|_{C}} \rightarrow \begin{aligned}
& \oplus\left(T_{y_{i}} S \otimes T_{y_{i}} D_{i}\right) \\
& \oplus\left(T_{z_{j}} S \otimes T_{z_{j}} G_{j}\right)
\end{aligned} \rightarrow 0 .
$$

We can move the $T^{0}$ in this sequence from the second to the fourth term since the bundle $N_{\rho^{\sharp}} \rightarrow \mathfrak{M}_{\rho^{\sharp}}$ splits off a trivial bundle with $T^{0}$ as the fiber. We then define the split injection $i$ using the diagram:

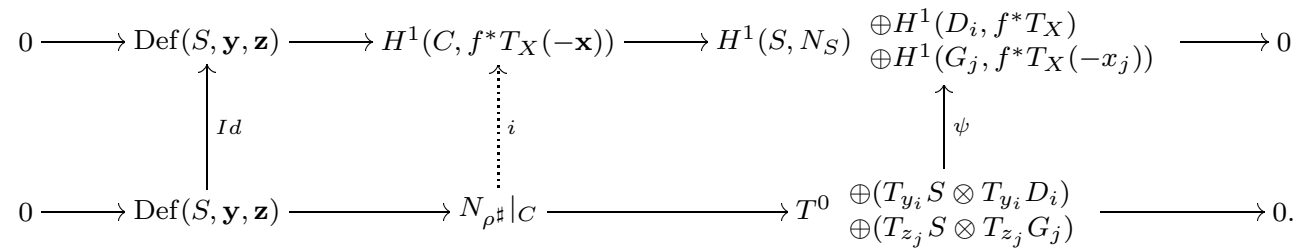

We will define $\psi$ as the sum of split injections

$$
\begin{aligned}
& \psi_{0}: T^{0} \rightarrow H^{1}\left(S, N_{S}\right), \\
& \psi_{j}: T_{z_{j}} S \otimes T_{z_{j}} G_{j} \rightarrow H^{1}\left(G_{j}, f^{*} T_{X}\left(-x_{j}\right)\right), \\
& \psi_{i}: T_{y_{i}} S \otimes T_{y_{i}} D_{i} \rightarrow H^{1}\left(D_{i}, f^{*} T_{X}\right)
\end{aligned}
$$

in such a way that the induced map $i$ makes diagram (9) commute. The theorem will then follow.

The map $\psi_{0}$ is an isomorphism of lines (this is proven at the very end of the proof of Lemma [5.4).

To define the maps $\psi_{i}$ in a way compatible with diagram (9), we should recall how the various maps and spaces involved are defined. The space $T_{y_{i}} S \otimes T_{y_{i}} D_{i}$ classifies infinitesimal deformations of the node at $y_{i}$. We find that what we seek is a split injection $\psi$ making the following diagram commute (we've dropped the $i$ from the notation):

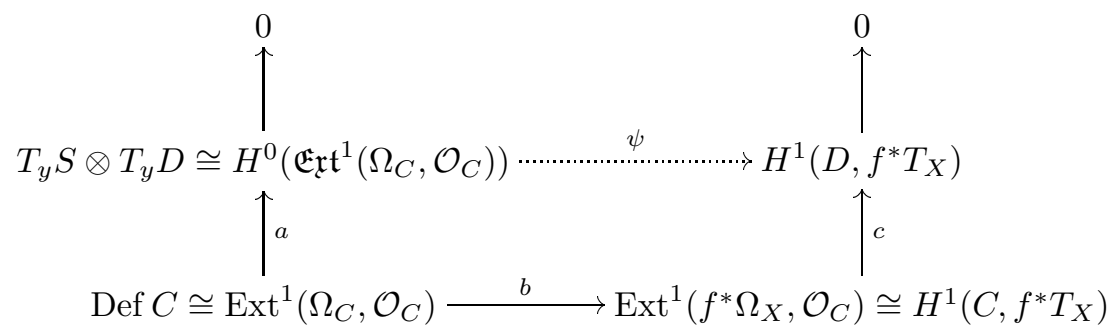


where the map $a$ comes from the local-to-global spectral sequence for Ext, $b$ is induced by $f^{*} \Omega_{X} \rightarrow \Omega_{C}$, and $c$ is induced by the restriction $\mathcal{O}_{C} \rightarrow \mathcal{O}_{D}$.

The map $\psi$ exists if and only if $c \circ b$ is 0 on Ker $a$. This is true and follows from stability of the map $f: C \rightarrow X$.

The dual map

$$
\psi^{\vee}: H^{1}\left(D, f^{*} T_{X}\right)^{\vee} \cong H^{0}\left(D, f^{*} \Omega_{X} \otimes \omega_{D}\right) \rightarrow \Omega_{y} S \otimes \Omega_{y} D
$$

can be identified as follows. Since $\left.\left(f^{*} \Omega_{X} \otimes \omega_{D}\right)\right|_{y}=\Omega_{y} X \otimes \Omega_{y} D$ and $\Omega_{y} X=$ $\Omega_{y} S \oplus \Omega_{y} N$, we obtain $\psi^{\vee}$ by restriction and then projection onto the first factor.

The cohomology sequence associated to the short exact sequence

$$
0 \rightarrow f^{*} \Omega_{X} \otimes \omega_{D}(-y) \rightarrow f^{*} \Omega_{X} \otimes \omega_{D} \rightarrow \Omega_{y} X \otimes \Omega_{y} D \rightarrow 0
$$

has the terms

$H^{0}\left(D, f^{*} \Omega_{X} \otimes \omega_{D}\right) \rightarrow\left(\Omega_{y} S \otimes \Omega_{y} D\right) \oplus\left(\Omega_{y} N \otimes \Omega_{y} D\right) \rightarrow H^{1}\left(D, f^{*} \Omega_{X} \otimes \omega_{D}(-y)\right)$,

so to see that $\psi^{\vee}$ is surjective we just need to show that

$$
\Omega_{y} S \otimes \Omega_{y} D \rightarrow H^{1}\left(D, f^{*} \Omega_{X} \otimes \omega_{D}(-y)\right)
$$

is 0 .

Recall that $\left.f\right|_{D \cup S}$ factors as $g \circ h$ where $h$ is a local embedding $h: \Sigma(a) \rightarrow$ $N \cup S \subset X$ and so $\left.f\right|_{D}$ factors as $g^{\prime} \circ h^{\prime}$ where $h^{\prime}: \Sigma^{\prime} \rightarrow N \subset X$ and $\Sigma^{\prime}$ is a linear chain of rational curves. We will drop the primes from the sequel. Since $h$ is a local embedding of a local complete intersection, the conormal bundle to the map $N_{h}^{\vee}$ is a line bundle. It fits into the exact sequence:

$$
0 \rightarrow N_{h}^{\vee} \rightarrow h^{*} \Omega_{X} \rightarrow \Omega_{\Sigma} \rightarrow 0 .
$$

The map $\Omega_{y} S \otimes \Omega_{y} D \rightarrow H^{1}\left(f^{*} \Omega_{X} \otimes \omega_{D}(-y)\right)$ factors through the map

$$
H^{1}\left(g^{*} N_{h}^{\vee} \otimes \omega_{D}(-y)\right) \rightarrow H^{1}\left(f^{*} \Omega_{X} \otimes \omega_{D}(-y)\right)
$$

since $\left.N_{h}^{\vee}\right|_{y}=\Omega_{y} S$. But since $N_{h}^{\vee}$ is degree 2 restricted to each component of $\Sigma$, the pull back of the dual $g^{*} N_{h}$ is non-positive on each component and has degree smaller than -2 on at least one component. Thus we have that

$$
H^{1}\left(D, g^{*} N_{h}^{\vee} \otimes \omega_{D}(-y)\right)=H^{0}\left(D, g^{*} N_{h}(y)\right)^{\vee}=0
$$

and so $\psi^{\vee}$ is surjective.

To see that $\psi^{\vee}$ is split we need to exhibit a subline of $H^{0}\left(f^{*} \Omega_{X} \otimes \omega_{D}\right)$ mapping onto $\Omega_{y} S \otimes \Omega_{y} D$. Consider the map

$$
d f: f^{*} \Omega_{X} \rightarrow \Omega_{D}
$$

as an element of $H^{0}\left(f^{*} T_{X} \otimes \Omega_{D}\right)$. Since $X$ is a $K 3$ surface we have an isomorphism $\Omega_{X} \cong T_{X}$ and so $d f$ gives us an element of $H^{0}\left(f^{*} \Omega_{X} \otimes \Omega_{D}\right)$ and consequently an element of $H^{0}\left(f^{*} \Omega_{X} \otimes \omega_{D}\right)$ (which we still call $d f$ ). Evaluation at $y$ determines an isomorphism of the line spanned by $d f$ with $\Omega_{y} S \otimes \Omega_{y} D$ and so $\psi^{\vee}$ splits.

The case of $\psi_{j}$ can be handled in a similar (but easier) fashion.

Corollary B.2. The splitting $H^{1}\left(C, f^{*} T_{X}(-\mathbf{x})\right) \rightarrow N_{\rho^{\sharp}}$ provides the map $\theta$ needed in the hypothesis of Proposition A.1] of Appendix $A$ in the setting of this paper (cf. Remark A.3). 


\section{REFERENCES}

[1] W. Barth, C. Peters, and A. Van de Ven. Compact Complex Surfaces, volume 4 of Ergebnisse der Mathematik und ihrer Grenzgebiete. Springer-Verlag, 1984. IMR 86c:32026

[2] Victor Batyrev. On the Betti numbers of birationally isomorphic projective varieties with trivial canonical bundles. Preprint, alg-geom//9710020.

[3] A. Beauville. Counting rational curves on K3 surfaces. Preprint alg-geom/9701019, 1997.

[4] K. Behrend. Personal communication, 1998.

[5] K. Behrend. Gromov-Witten invariants in algebraic geometry. Invent. Math., 127(3):601-617, 1997. MR 98i:14015

[6] K. Behrend and B. Fantechi. The intrinsic normal cone. Invent. Math., 128(1):45-88, 1997. MR 98e:14022

[7] K. Behrend and Yu. Manin. Stacks of stable maps and Gromov-Witten invariants. Duke Math. J., 85(1):1-60, 1996. MR 98i:14014

[8] M. Bershadsky, C. Vafa, and V. Sadov. D-branes and topological field theories. Nuclear Phys. B, 463(2-3):420-434, 1996. MR 97h:81213

[9] A. Besse. Einstein Manifolds, volume 10 of Ergebnisse der Mathematik und ihrer Grenzgebiete. Springer-Verlag, 1987. MR 88f:53087

[10] Xi Chen. Counting curves on K3. Ph.D. thesis, Harvard, 1997.

[11] Xi Chen. Singularities of rational curves on K3 surfaces. Preprint, math.AG/9812050, 1998.

[12] Xi Chen. Personal communication, 1999.

[13] David A. Cox and Sheldon Katz. Mirror Symmetry and Algebraic Geometry. American Mathematical Society, Providence, RI, 1999. CMP 99:09

[14] S. K. Donaldson. Yang-Mills invariants of 4-manifolds. In S. K. Donaldson and C. B. Thomas, editors, Geometry of Low-Dimensional Manifolds: Gauge Theory and Algebraic Surfaces, number 150 in London Mathematical Society Lecture Note Series. Cambridge University Press, 1989. MR 93f:57040

[15] B. Fantechi, L. Göttsche, and D. van Straten. Euler number of the compactified Jacobian and multiplicity of rational curves. J. Algebraic Geom., 8(1):115-133, 1999. MR 99i:14065

[16] R. Friedman and J. Morgan. Smooth Four-manifolds and Complex Surfaces, volume 27 of Ergebnisse der Mathematik und ihrer Grenzgebiete. Springer-Verlag, 1994. MR 95m:57046

[17] Alexander Givental. Stationary phase integrals, quantum Toda lattices, flag manifolds and the mirror conjecture. (alg-geom/9612001).

[18] Lothar Göttsche. A conjectural generating function for numbers of curves on surfaces. Comm. Math. Phys., 196(3):523-533, 1998. CMP 99:01

[19] L. Göttsche. The Betti numbers of the Hilbert scheme of points on a smooth projective surface. Math. Ann., pages 193-207, 1990. MR 91h:14007

[20] L. Göttsche and R. Pandharipande. The quantum cohomology of blow-ups of $\mathbf{P}^{2}$ and enumerative geometry. J. Differential Geom., 48(1):61-90, 1998. MR 99d:14057

[21] T. Graber and R. Pandharipande. Localization of virtual classes. Invent. Math., 135(2):487518, 1999. CMP 99:07

[22] Robin Hartshorne. Residues and duality. Springer-Verlag, Berlin, 1966. Lecture notes of a seminar on the work of A. Grothendieck, given at Harvard 1963/64. With an appendix by P. Deligne. Lecture Notes in Mathematics, No. 20. MR 36:5145

[23] Daniel Huybrechts. Compact hyper-Kähler manifolds: basic results. Invent. Math., 135(1):63113, 1999. CMP 99:06

[24] Andrew Kresch. Cycle groups for Artin stacks. math.AG/9810166.

[25] P. B. Kronheimer. Some non-trivial families of symplectic structures. Preprint, 1997.

[26] Jun Li and Gang Tian. Comparison of the algebraic and the symplectic Gromov-Witten invariants. (alg-geom/9712035).

[27] Jun Li and Gang Tian. Virtual moduli cycles and Gromov-Witten invariants of algebraic varieties. J. Amer. Math. Soc., 11(1):119-174, 1998. [MR 99d:14011

[28] Jun Li and Gang Tian. Virtual moduli cycles and Gromov-Witten invariants of general symplectic manifolds. In Topics in symplectic 4-manifolds (Irvine, CA, 1996), pages 47-83. Internat. Press, Cambridge, MA, 1998. CMP 98:17

[29] T.J. Li and A. Liu. Family Seiberg-Witten invariant. Preprint., 1997.

[30] Bong H. Lian, Kefeng Liu, and Shing-Tung Yau. Mirror principle. I. Asian J. Math., 1(4):729763, 1997. MR 99e:14062 
[31] Y. Ruan and G. Tian. A mathematical theory of quantum cohomology. J. Differential Geometry, 42(2), 1995. MR 96m:58033

[32] Yongbin Ruan. Virtual neighborhoods and pseudo-holomorphic curves. Preprint alggeom/9611021., 1996.

[33] Bernd Siebert. Gromov-Witten invariants of general symplectic manifolds. Preprint math.DG/9608105., 1996.

[34] Bernd Siebert. Algebraic and symplectic Gromov-Witten invariants coincide. Preprint math.AG/9804108., 1998.

[35] Charles A. Weibel. An introduction to homological algebra. Cambridge University Press, Cambridge, 1994. MR 95f:18001

[36] S.-T. Yau. On the Ricci curvature of a compact Kähler manifold and the complex Monge Ampère equation I. Comm. Pure and Appl. Math, 31:339-411, 1978. MR 81d:53045

[37] S.-T. Yau and E. Zaslow. BPS states, string duality, and nodal curves on K3. Nuclear Physics B, 471(3):503-512, 1996. Also: hep-th/9512121. MR 97e:14066

Department of Mathematics, Tulane University, 6823 St. Charles Ave., New Orleans, LOUISIANA 70118

E-mail address: jbryan@math.tulane.edu

School of Mathematics, University of Minnesota, Minneapolis, Minnesota 55455

E-mail address: leung@math.umn.edu 